\title{
High expression levels of pyrimidine metabolic rate-limiting enzymes are adverse prognostic factors in lung adenocarcinoma: a study based on The Cancer Genome Atlas and Gene Expression Omnibus datasets
}

\author{
Haiwei Wang ${ }^{1,2}$ (D) Xinrui Wang ${ }^{1,2} \cdot$ Liangpu Xu $^{1,2} \cdot$ Ji Zhang $^{3} \cdot$ Hua Cao ${ }^{1,2}$
}

Received: 24 March 2020 / Accepted: 25 June 2020 / Published online: 8 July 2020

(C) The Author(s) 2020

\begin{abstract}
Reprogramming of metabolism is described in many types of cancer and is associated with the clinical outcomes. However, the prognostic significance of pyrimidine metabolism signaling pathway in lung adenocarcinoma (LUAD) is unclear. Using the Gene Expression Omnibus (GEO) and The Cancer Genome Atlas (TCGA) datasets, we found that the pyrimidine metabolism signaling pathway was significantly enriched in LUAD. Compared with normal lung tissues, the pyrimidine metabolic rate-limiting enzymes were highly expressed in lung tumor tissues. The high expression levels of pyrimidine metabolic-rate limiting enzymes were associated with unfavorable prognosis. However, purinergic receptors P2RX1, P2RX7, P2RY12, P2RY13, and P2RY14 were relatively downregulated in lung cancer tissues and were associated with favorable prognosis. Moreover, we found that hypo-DNA methylation, DNA amplification, and TP53 mutation were contributing to the high expression levels of pyrimidine metabolic rate-limiting enzymes in lung cancer cells. Furthermore, combined pyrimidine metabolic rate-limiting enzymes had significant prognostic effects in LUAD. Comprehensively, the pyrimidine metabolic rate-limiting enzymes were highly expressed in bladder cancer, breast cancer, colon cancer, liver cancer, and stomach cancer. And the high expression levels of pyrimidine metabolic rate-limiting enzymes were associated with unfavorable prognosis in liver cancer. Overall, our results suggested the mRNA levels of pyrimidine metabolic rate-limiting enzymes CAD, DTYMK, RRM1, RRM2, TK1, TYMS, UCK2, NR5C2, and TK2 were predictive of lung cancer as well as other cancers.
\end{abstract}

Keywords Lung adenocarcinoma · Pyrimidine metabolic rate-limiting enzymes · Purinergic receptors · Gene expression omnibus · The Cancer genome atlas

Haiwei Wang and Xinrui Wang contributed equally to this work.

Electronic supplementary material The online version of this article (https://doi.org/10.1007/s11302-020-09711-4) contains supplementary material, which is available to authorized users.

Haiwei Wang

hwwang@sibs.ac.cn

Ji Zhang

Zj11222@rjh.com.cn

$\triangle$ Hua Cao

caohua69@fjmu.edu.cn
1 Fujian Key Laboratory for Prenatal Diagnosis and Birth Defect, Fujian Maternity and Child Health Hospital,, Affiliated Hospital of Fujian Medical University, Fuzhou, Fujian, China

2 Key Laboratory of Technical Evaluation of Fertility Regulation for Non-human Primate, National Health and Family Planning Commission, Fuzhou, Fujian, China

3 State Key Laboratory for Medical Genomics, Shanghai Institute of Hematology, Rui-Jin Hospital Affiliated to School of Medicine, Shanghai Jiao Tong University, Shanghai, China 


\section{Background}

Lung cancer is one of the most commonly diagnosed cancer and the leading cause of cancer-related mortality [1-3]. Although some improvements of treatment have been achieved in the past few decades, the 5-year survival rate of lung cancer patients is still low $[4,5]$. Lung cancer is a heterogeneous disease, including small cell lung cancer and non-small cell lung cancer (NSCLC) [6]. NSCLC accounts for the $85 \%$ of lung cancer cases and could be further divided into 3 major pathologic subtypes: lung adenocarcinoma (LUAD), lung squamous cell carcinoma (LUSC), and large-cell carcinoma [7]. Each subtype of NSCLC demonstrates different molecular profiles and different drug response [8,9]. Although gene alterations $[10,11]$, mRNA expression signature $[12,13]$, microRNA profiles [14, 15], long non-coding RNAs [16, 17], immune signature [18], and tumor microenvironment [19] are used for the prognosis of NSCLC, more candidate biomarkers are needed.

Reprogramming of cell metabolism is a hallmark of cancer [20]. Cancer cells increase glucose uptake and utilize aerobic glycolysis to facilitate the uncontrolled cell proliferation [21]. Glycolysis-related gene signature is associated with the overall survival of LUAD patients [22]. Besides the misregulation of glucose metabolism, the pyrimidine metabolism is also disrupted during the development of cancer [23]. The disruption of the pyrimidine metabolism is reflected by the malfunctions of the pyrimidine metabolic rate-limiting enzymes. The high expression levels of pyrimidine metabolic rate-limiting enzymes CAD, CTPS, CTPS2, DHODH, DTYMK, NT5C2, NT5C3, RRM1, RRM2, TK1, TK2, TYMS, UCK2, and UCKL1 are illustrated in poorly differentiated liver cancer patients and correlated poor clinical outcomes [24]. Inhibition of pyrimidine synthesis by targeting pyrimidine metabolic rate-limiting enzymes DHODH and CAD could accentuate the molecular therapy response in glioblastoma [25]. Also, inhibition of pyrimidine synthesis sensitizes triple-negative breast cancer cells to chemotherapy [26]. However, the prognostic significance of the pyrimidine metabolism signaling pathway in LUAD is unclear.

In the present study, we used large cohorts of lung cancer patients derived from Gene Expression Omnibus (GEO) and The Cancer Genome Atlas (TCGA) datasets to demonstrate the prognostic significance of pyrimidine metabolic ratelimiting enzymes and purinergic receptors in LUAD. Overall, the analysis of GEO and TCGA datasets allowed an improved understanding of the functions of pyrimidine metabolic rate-limiting enzymes and purinergic receptors. The results also indicated the potential biomarkers of the pyrimidine metabolic rate-limiting enzymes for further clinical studies.

\section{Methods}

\section{Data collection}

The TCGA LUAD and LUSC gene expression, DNA mutation, and DNA methylation, along with the clinical datasets, were downloaded from the TCGA hub (https:// tcga.xenahubs.net). The LUAD and LUSC gene expression data was generated from RNA-seq and the DNA methylation data was generated from Illumina HumanMethylation450 Bead Chip. Gene expression data derived from bladder urothelial carcinoma (BLCA), breast invasive carcinoma (BRCA), colon adenocarcinoma (COAD), esophageal carcinoma (ESCA), head and neck squamous cell carcinoma (HNSC), kidney renal clear cell carcinoma (KIRC), kidney renal papillary cell carcinoma (KIRP), liver hepatocellular carcinoma (LIHC) , stomach adenocarcinoma (STAD), and thyroid cancer (THCA) were also downloaded from TCGA hub.

The gene expression series matrix of normal and cancerous lung tissues was downloaded from the GEO website (www. ncbi.nlm.nih.gov/geo) and included GSE7670, GSE10072, GSE18842, GSE19188, GSE27262, GSE30219, GSE31210, GSE31908, GSE33532, and GSE75324 datasets. The DNA methylation data of patients with LUAD was downloaded from the GEO datasets with GEO number GSE32867 and GSE62948. All the GEO expression datasets were based on Affymetrix Human Genome microarray.

Clinical and raw data of MSKCC dataset are downloaded from http://cbio.mskcc.org/Public/lung_array_data/ [27]. The detailed description of the collected data used in this study is illustrated in Fig. 1a.

\section{Gene Expression Omnibus data processing}

The GEO expression datasets were processed using R software (version 3.5.0, https://www.r-project.org/). The matrix file of each dataset was annotated with corresponding platform. When multiple probes corresponded to the same gene symbol, the expression values were averaged using "plyr" package (version 1.8.5) in R software. Plyr package includes multiple tools for splitting, applying, and combining data and could be downloaded from bioconductor (https://cran.r-project. org/web/packages/plyr/index.html). The different gene expression between normal and lung cancer samples was determined using paired Student's $t$ test. The different DNA methylation intensity between normal and lung cancer samples was also determined using paired Student's $t$ test.

\section{Gene set enrichment analysis}

The metabolic singling pathways enriched in lung cancer gene expression profiling were determined using Gene 
a

\begin{tabular}{lllll}
\hline GSE number Normal & Cancer & Cancer subtype & Platform \\
\hline GSE7670 & $\mathrm{N}=26$ & $\mathrm{~N}=26$ & Lung adenocarcinoma & Affymetrix Human Genome U133A Array \\
GSE10072 & $\mathrm{N}=49$ & $\mathrm{~N}=58$ & Lung adenocarcinoma & Affymetrix Human Genome U133A Array \\
GSE18842 & $\mathrm{N}=45$ & $\mathrm{~N}=45$ & Lung adenocarcinoma & Affymetrix Human Genome U133 Plus 2.0 Array \\
GSE19188 & $\mathrm{N}=65$ & $\mathrm{~N}=91$ & Non-small cell lung cancer & Affymetrix Human Genome U133 Plus 2.0 Array \\
GSE27262 & $\mathrm{N}=25$ & $\mathrm{~N}=25$ & Lung adenocarcinoma & Affymetrix Human Genome U133 Plus 2.0 Array \\
GSE30219 & $\mathrm{N}=14$ & $\mathrm{~N}=293$ & Unclassified lung cancer & Affymetrix Human Genome U133 Plus 2.0 Array \\
GSE31210 & $\mathrm{N}=20$ & $\mathrm{~N}=226$ & Lung adenocarcinoma & Affymetrix Human Genome U133 Plus 2.0 Array \\
GSE31908 & $\mathrm{N}=20$ & $\mathrm{~N}=30$ & Lung adenocarcinoma & Affymetrix Human Genome U133A Array \\
GSE33532 & $\mathrm{N}=20$ & $\mathrm{~N}=80$ & Non-small cell lung cancer & Affymetrix Human Genome U133 Plus 2.0 Array \\
GSE75324 & $\mathrm{N}=52$ & $\mathrm{~N}=80$ & Unclassified lung cancer & Affymetrix Human Genome U133A Array \\
LUAD & $\mathrm{N}=57$ & $\mathrm{~N}=515$ & Lung adenocarcinoma & Illumina HiSeq200 RNA-Seq \\
LUSC & $\mathrm{N}=51$ & $\mathrm{~N}=502$ & Lung Squamous Cell Carcinoma & Illumina HiSeq200 RNA-Seq \\
GSE32867 & $\mathrm{N}=59$ & $\mathrm{~N}=59$ & Lung adenocarcinoma & Illumina Human Methylation27 BeadChip \\
GSE62948 & $\mathrm{N}=28$ & $\mathrm{~N}=28$ & Lung adenocarcinoma & Illumina Human Methylation27 BeadChip \\
LUAD & $\mathrm{N}=29$ & $\mathrm{~N}=458$ & Lung adenocarcinoma & Illumina Human Methylation450 BeadChip \\
MSKCC & $\mathrm{N}=0$ & $\mathrm{~N}=107$ & Lung adenocarcinoma & Affymetrix Human Genome U133A Array \\
\hline GSE number & $\mathrm{TP53}$ mutant & $\mathrm{TP53}$ wild type & Cancer subtype & \\
\hline GSE72094 & $\mathrm{N}=111$ & $\mathrm{~N}=331$ & Lung adenocarcinoma & Affymetrix 2.0 microarray \\
\hline
\end{tabular}

b

Enrichment plots of pyrimidine metabolism signaling pathway

GSE7670

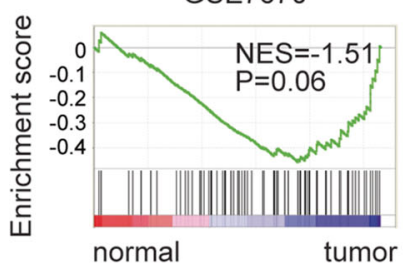

GSE27262

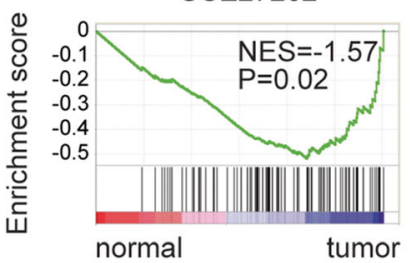

GSE33532

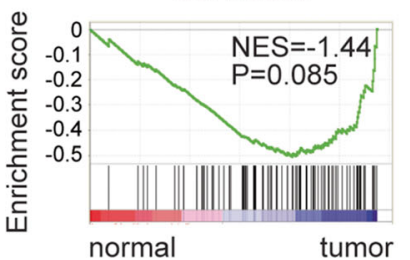

GSE10072

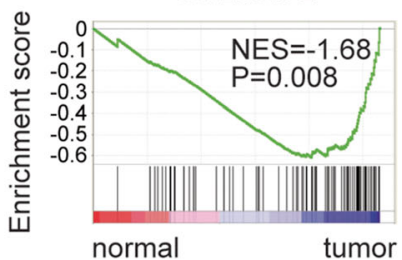

GSE30219

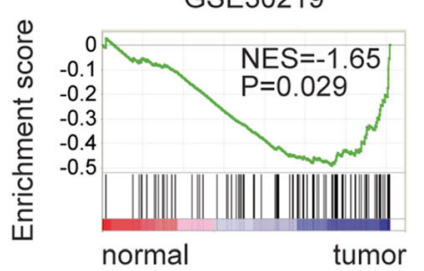

GSE75324

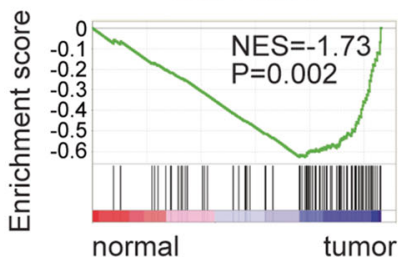

Fig. 1 Pyrimidine metabolism signaling pathway is highly enriched in lung cancer. a Table showed the detailed GEO datasets and TCGA datasets used in this study. b Enrichment plots demonstrated the enriched pyrimidine metabolism signaling pathway in GSE7670, GSE10072, GSE18842, GSE19188, GSE27262, GSE30219,

set enrichment analysis (GSEA) software (version 2.0) [28]. The GSEA software and the signaling pathways gene sets were downloaded from the GSEA website (www.broad.mit.edu/gsea/index.html). Genes were
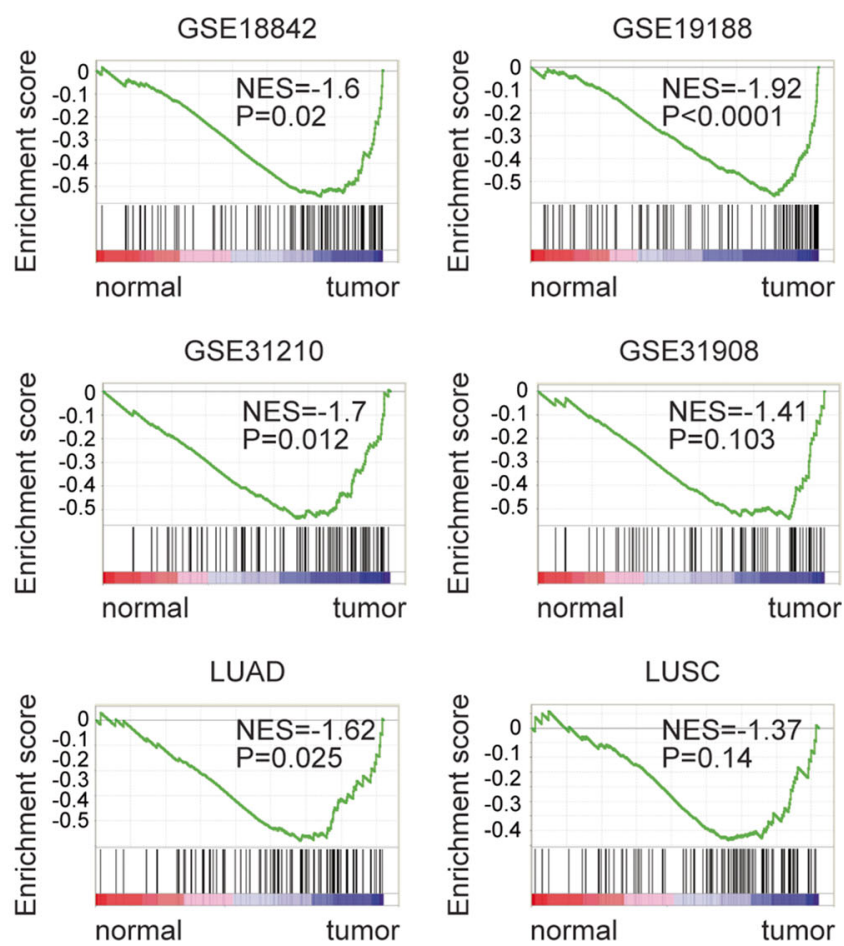

GSE31210, GSE31908, GSE33532, GSE75324, and TCGA LUAD, LUSC datasets. Enrichment of normalized enrichment score (NES) and $P$ values were presented. LUAD: lung adenocarcinoma; LUSC: lung squamous cell carcinoma;

ranked by the signal-to-noise ratio, and statistical significance was determined by 1000 gene set permutations. The results of significance should meet the criteria of nominal $P$ value less than 0.05 . 


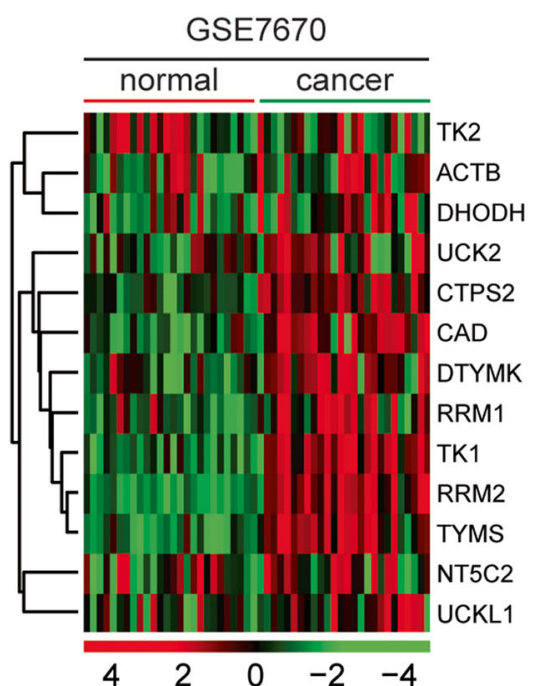

GSE19188

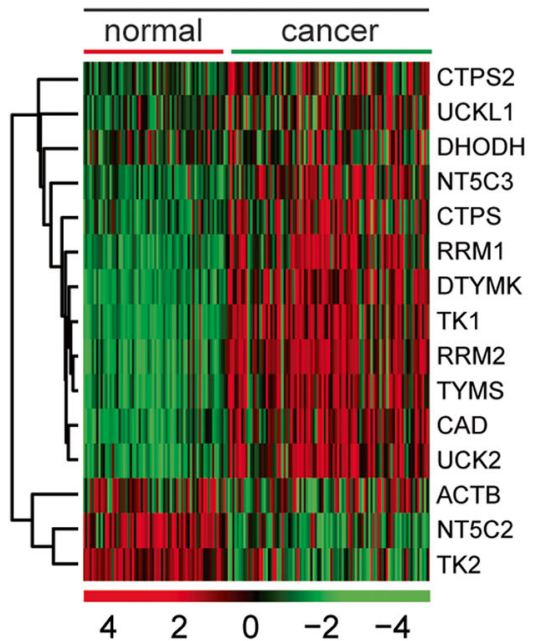

GSE33532

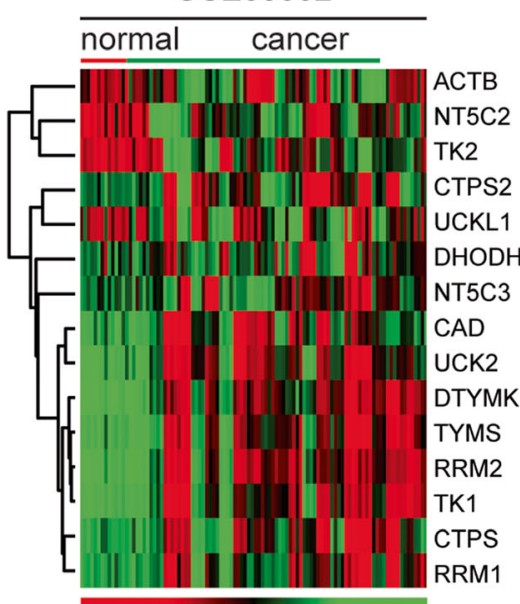

$\begin{array}{lllll}4 & 2 & 0 & -2 & -4\end{array}$
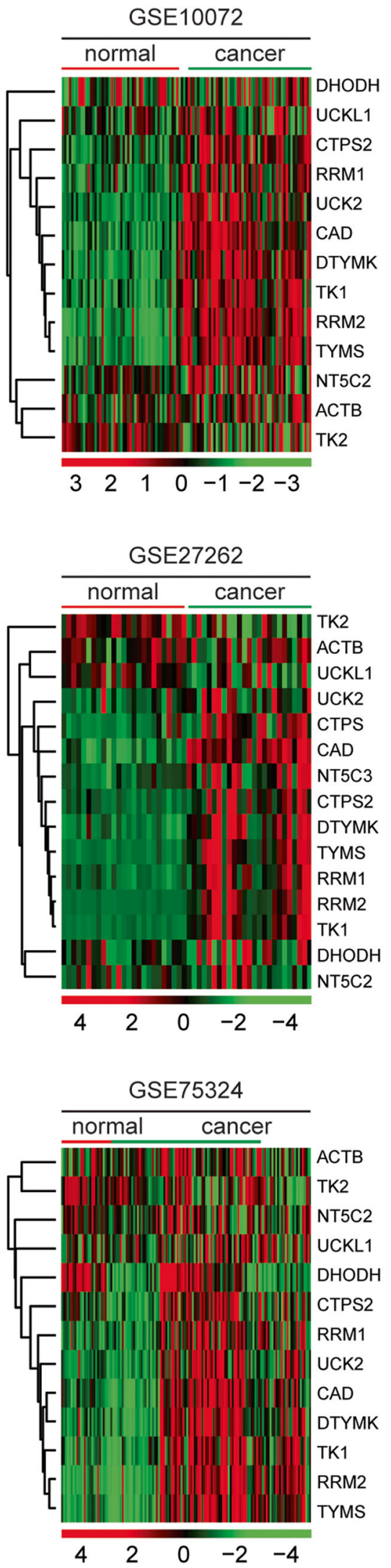
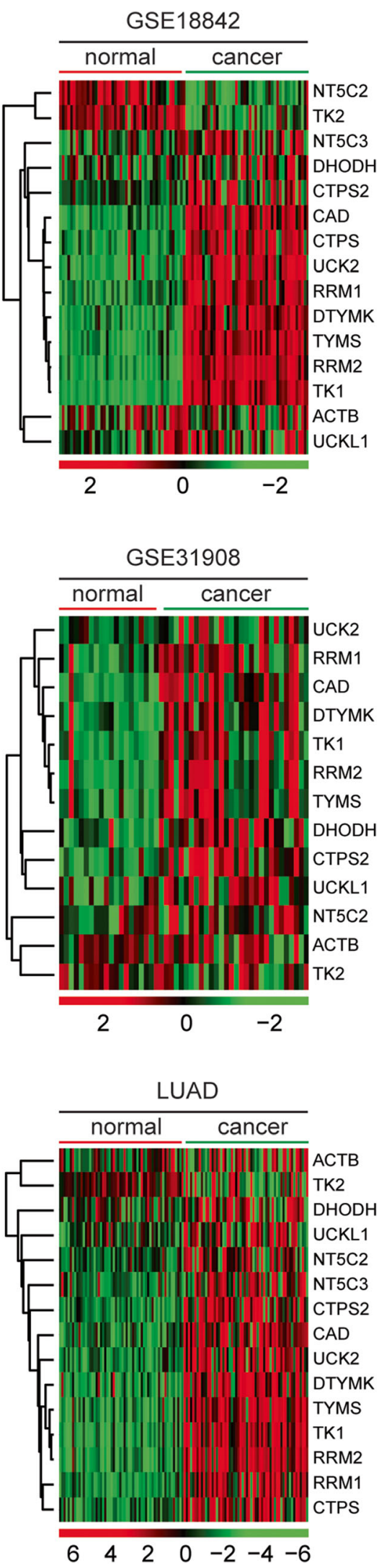
Fig. 2 Pyrimidine metabolic rate limiting enzymes are up-regulated in lung cancer cells across different datasets. Heatmaps demonstrated the expression levels of pyrimidine metabolic rate-limiting enzymes in normal lung tissues and lung cancer tissues in GSE7670, GSE10072, GSE1 8842, GSE19188, GSE27262, GSE31908, GSE33532, GSE75324, and TCGA LUAD datasets. Upregulated (red), downregulated (green), and unchanged (black) genes were delineated. The expression levels of $\beta$-actin (ACTB) in normal and cancer tissues were used as control

\section{Heatmap presentation}

Heatmaps were created by R software "pheatmap" package (version 1.0.12). The pheatmap package and the basic usage were downloaded from bioconductor (https://cran.rproject.org/web/packages/pheatmap/). The clustering scale was determined by the "average" method. The clustering distance was determined by the "correlation" method. Other parameters were provided in the usage of the pheatmap.

\section{Survival analysis using Gene Expression Omnibus dataset}

The Kaplan-Meier plotter (https://kmplot.com/analysis/) [29, 30] was used to identify the association between the expression levels of the pyrimidine metabolic ratelimiting enzymes, purinergic receptors, and overall survival in lung cancer derived from GEO datasets. The Kaplan-Meier plotter is an online survival analysis tool to rapidly assess the prognostic effects of genes using GEO microarray data. The patients were divided by the auto select best cutoff using the expression of the pyrimidine metabolic rate-limiting enzymes. $P$ values were determined using Log-rank test.

\section{Survival analysis using The Cancer Genome Atlas dataset}

R statistics software "survival" package (version 3.1-8) was used to identify the clinical influence of pyrimidine metabolic rate-limiting enzymes on overall survival in patients derived from TCGA LUAD, LUSC, LIHC, BRCA, and STAD datasets. The survival package and the basic usage were downloaded from bioconductor (https://cran.r-project.org/ web/packages/survival/index.html). The patients were divided into two clusters based on the mean expression levels of genes. Kaplan-Meier estimator was applied to determine the clinical outcomes in patients with high expression levels and low expression levels of genes. $P$ values were determined using Log-rank test.
Oncoprints of the pyrimidine metabolic rate-limiting enzymes

The genomic alterations of pyrimidine metabolic rate-limiting enzymes in LUAD patients were downloaded from cbioportal (version 3.2.0) based on the TCGA datasets (http://www. cbioportal.org/index.do).

\section{Correlation plots of the pyrimidine metabolic rate- limiting enzymes}

Correlation plots of the pyrimidine metabolic rate-limiting enzymes were created using the "corrplot" package (version 0.84 ) in R. The corrplot package and the basic usage were downloaded from bioconductor (https://cran.r-project.org/ web/packages/corrplot/index.html). The Spearman's correlation test was used to demonstrate the correlation efficiency.

\section{Multivariate Cox regression}

Multivariate Cox regression was analyzed by "coxph" method in R software survival package (version 3.1-8). The survival package and the basic usage were downloaded from bioconductor (https://cran.r-project.org/web/packages/ survival/index.html). Log-rank test was used to calculate the $P$ values.

\section{Statistical analysis}

The box plots were generated from GraphPad Prism software (version 5.0). Statistical analysis was performed using the paired Student's $t$ test using R software. $P$ value less than 0.05 was chosen to be significantly different.

\section{Results}

\section{Pyrimidine metabolism signaling pathway is highly enriched in lung tumor samples across different datasets}

In order to reveal the metabolism-related transcriptional profiling in lung cancer, we analyzed lung cancer patients with expression data from previously published GEO datasets. Totally, 1290 samples were collected from ten previously published datasets based on Affymetrix gene microarray platforms, including 336 normal lung samples and 954 lung tumor samples. Most of the lung cancer patients belonged to LUAD subtype. A detailed description of the collected data used in this study is illustrated in Fig. 1a.

We then identified the enriched metabolic signaling pathways in patients with lung cancer using the GSEA assay. 

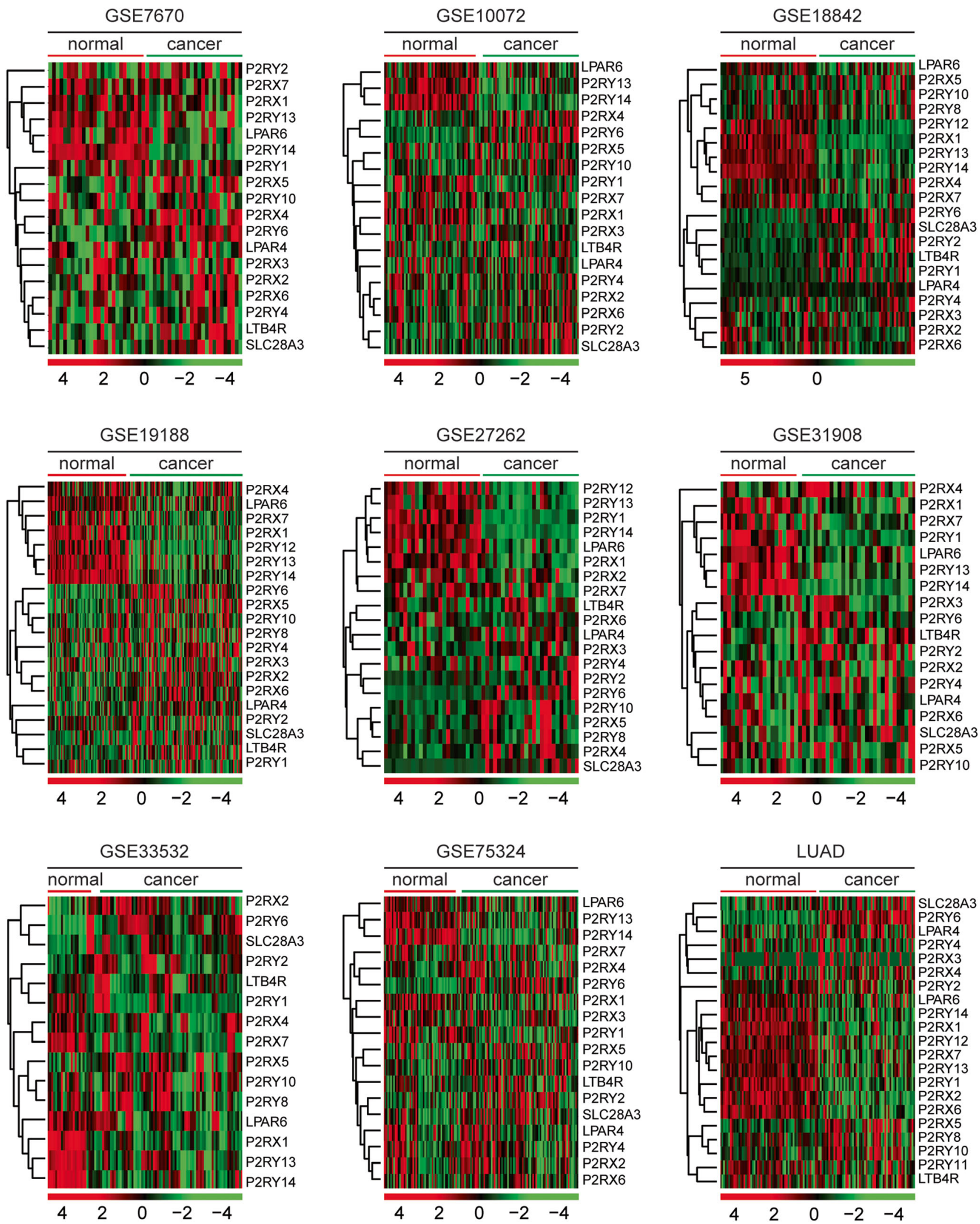

Fig. 3 The expression levels of purinergic receptors in lung cancer cells. Heatmaps demonstrated the expression levels of purinergic receptors in GSE18842, GSE19188, GSE27262, GSE31908, GSE33532, GSE75324, and TCGA LUAD datasets 
a

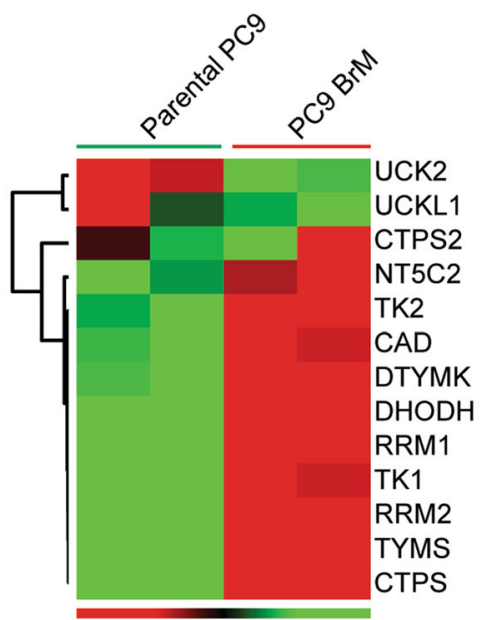

$\begin{array}{lllll}1 & 0.5 & 0 & -0.5 & -1\end{array}$ b

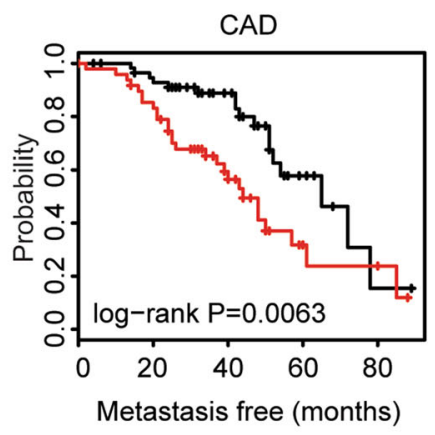

RRM2

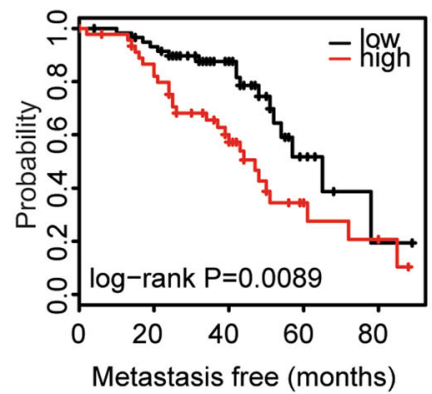

TK1

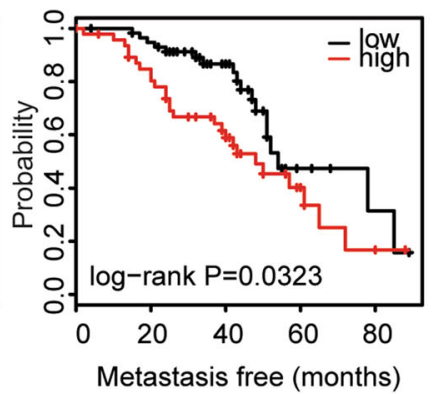

TYMS

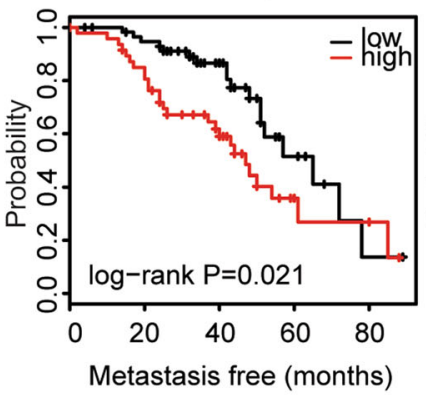

UCK2

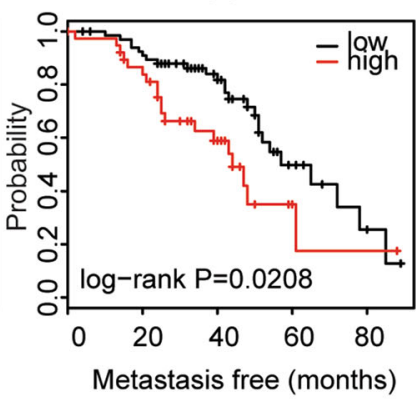

C

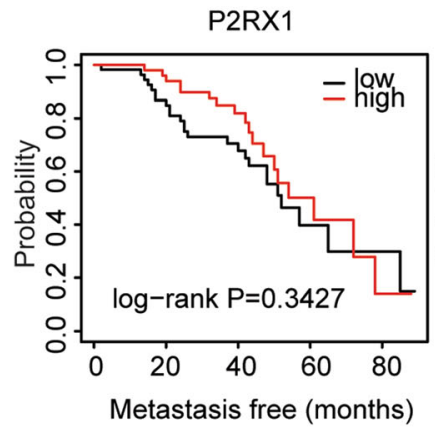

Fig. 4 Pyrimidine metabolic rate-limiting enzymes are upregulated in metastatic lung cancer cells and associated with lung cancer recurrence. a Heatmaps demonstrated the expression levels of pyrimidine metabolic rate-limiting enzymes in parental PC9 cells and PC9 BrM cells. b The Kaplan-Meier plotters demonstrated the associations between pyrimidine

Among all the enriched metabolic signaling pathways, the pyrimidine metabolism signaling pathway was significantly enriched in seven out of ten datasets, including GSE10072, GSE18842, GSE19188, GSE27262, GSE30219, GSE31210, and GSE75324 datasets, representing the most frequently enriched metabolic signaling pathway (Fig. 1b). Only in GSE7670, GSE31908, and GSE33532 three datasets, the
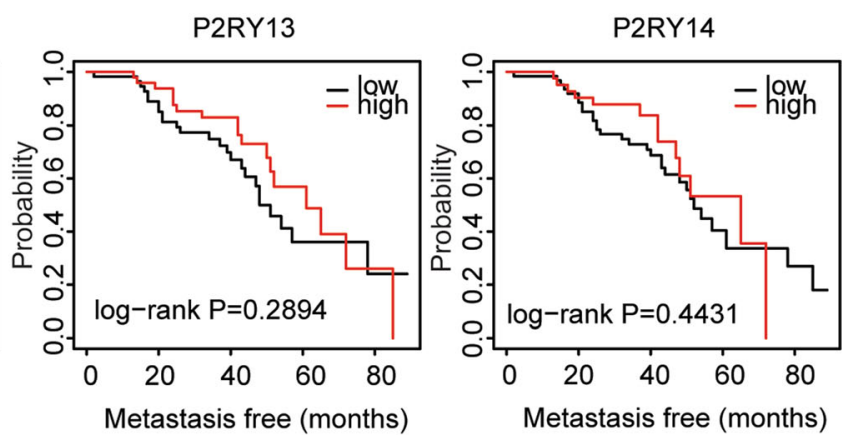

metabolic rate-limiting enzymes and lung cancer recurrence using the MSKCC dataset. The log-rank test was used to determine the overall survival $P$ value. c The Kaplan-Meier plotters demonstrated the associations between purinergic receptors P2RX1, P2RX2, P2RY13, and $\mathrm{P} 2 \mathrm{RY} 14$ and lung cancer recurrence using the MSKCC dataset

pyrimidine metabolism signaling pathway was not significantly correlated with the transcriptional profiling of lung cancer (Fig. 1b).

Using the TCGA lung cancer dataset, we found that the pyrimidine metabolism signaling pathway was positively associated with the transcriptional profiling of lung cancer in LUAD dataset (Fig. 1b). However, in another subtype of lung 
a

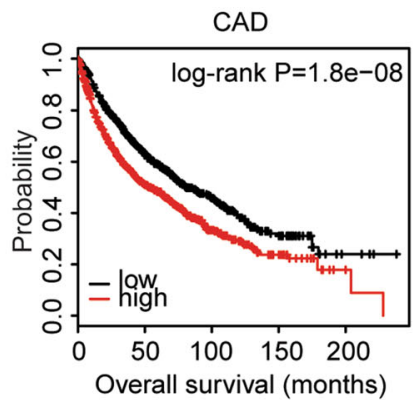

NT5C3B
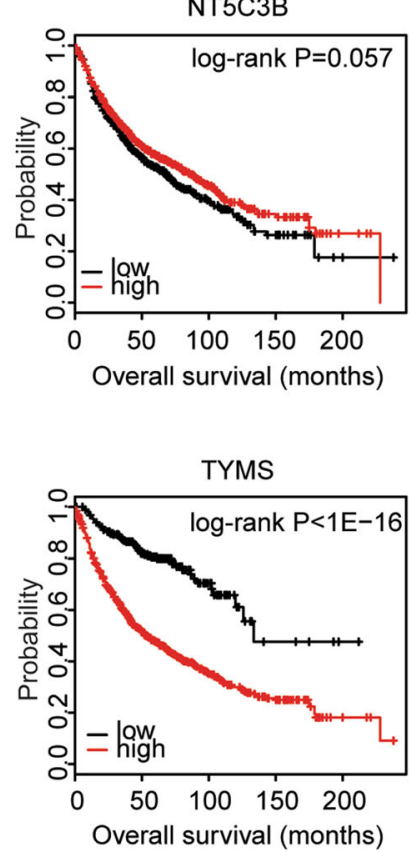

b
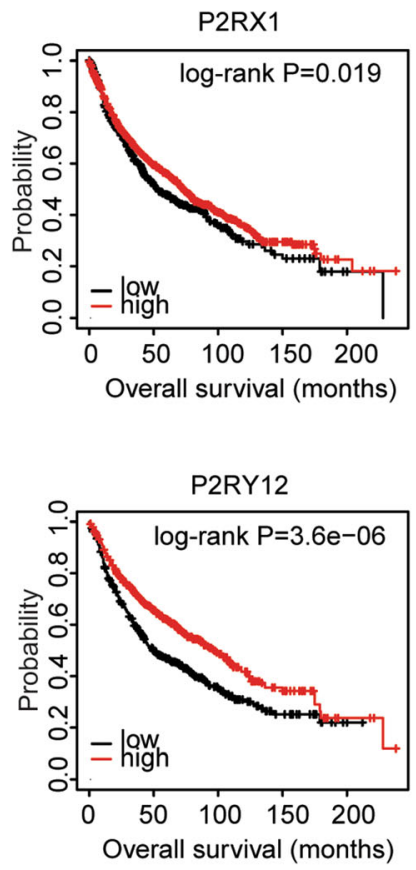

CTPS

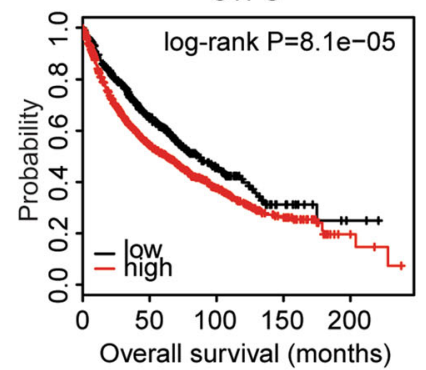

RRM1

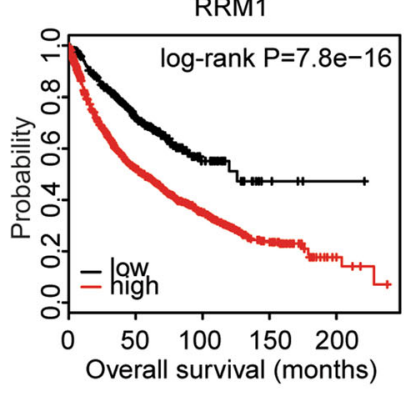

UCK2

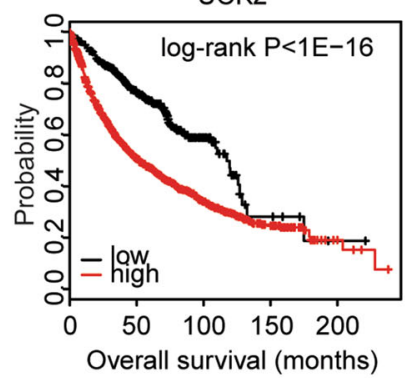

DHODH

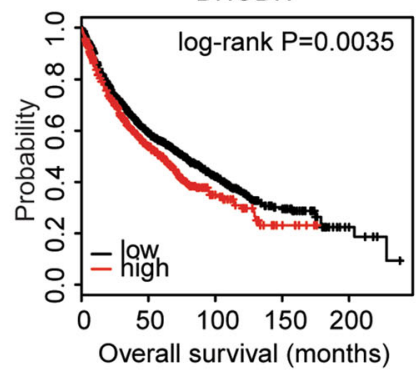

RRM2

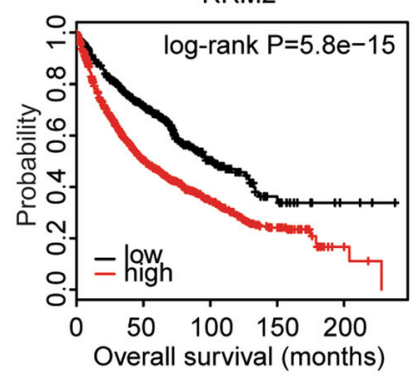

NR5C2

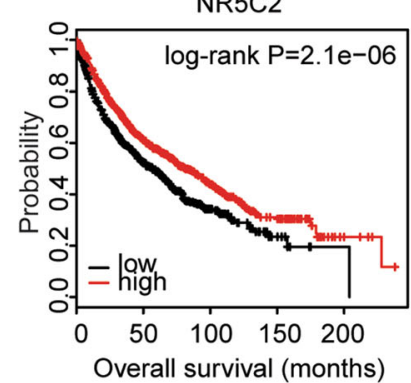

DTYMK

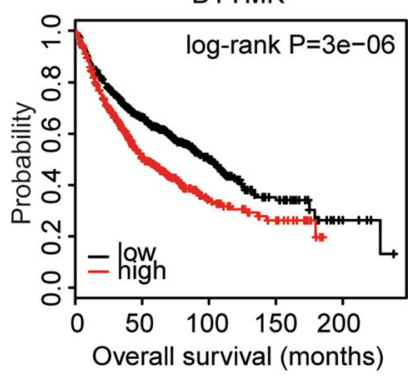

TK1

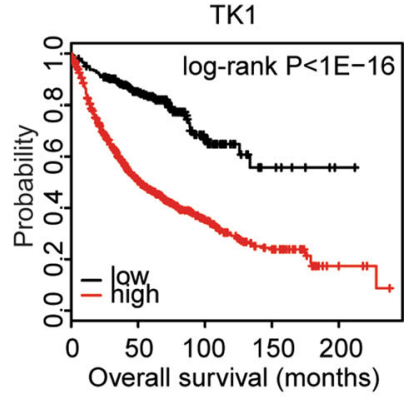

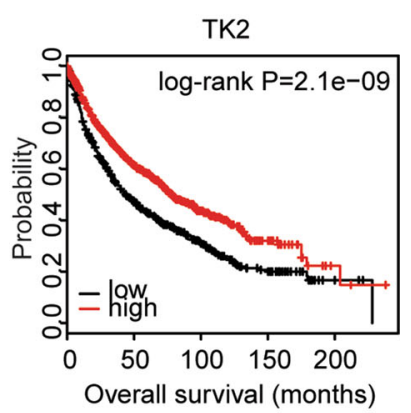
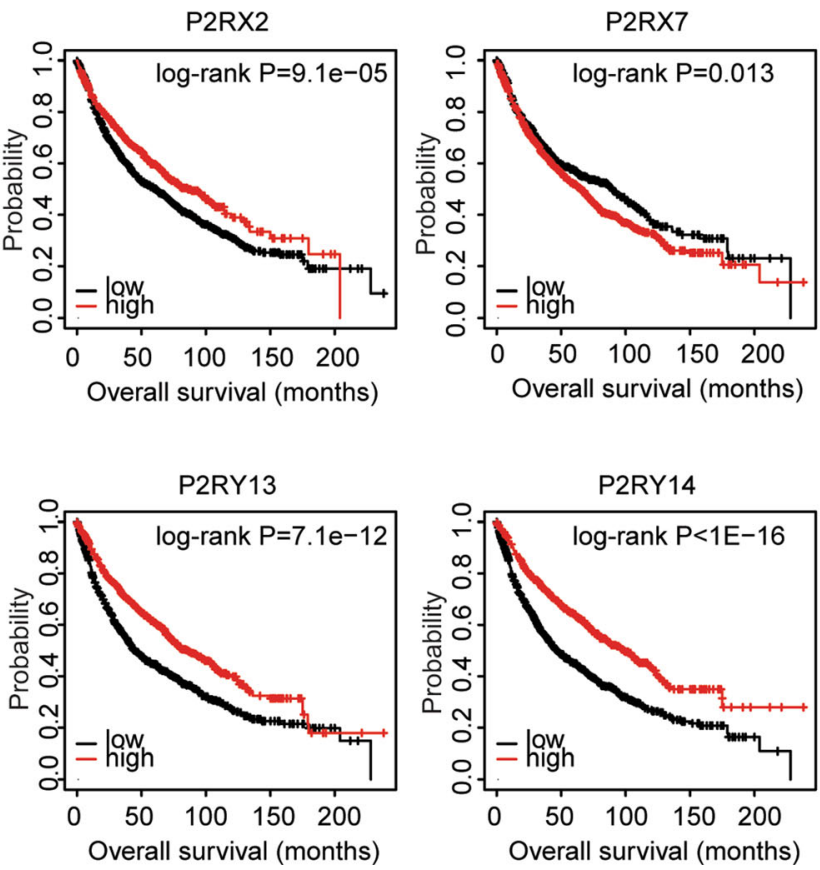

P2RY14

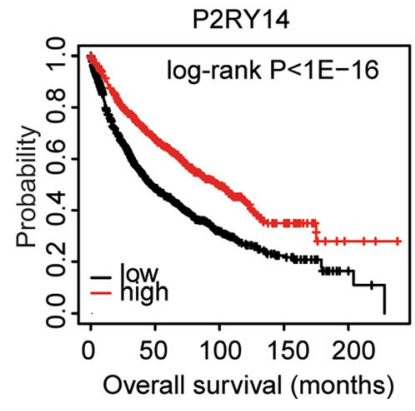


Fig. 5 Expression levels of pyrimidine metabolic rate limiting enzymes are associated with the tumor overall survival in lung cancer: analysis from GEO datasets. a The Kaplan-Meier plotters demonstrated the prognostic effects of the expression levels of pyrimidine metabolic ratelimiting enzymes TK1, UCK2, CAD, RRM1, RRM2, DTYMK, TYMS, TK2, and NR5C2 in lung cancer using the integrated GEO datasets. The patients were divided into two groups by the auto select best cutoff based on the expression levels of the pyrimidine metabolic rate-limiting enzymes. The log-rank test was used to determine the overall survival $P$ value. b The Kaplan-Meier plotters demonstrated the prognostic effects of the expression levels of purinergic receptors P2RX1, P2RX2, P2RX7, P2RY12, P2RY13, and P2RY14 in lung cancer

cancer LUSC, pyrimidine metabolism signaling pathway was not highly enriched (Fig. 1b).

\section{Pyrimidine metabolic rate-limiting enzymes are up- regulated in lung cancer cells across different datasets}

The pyrimidine metabolism signaling pathway was involving multiple genes. Previous results suggested that pyrimidine metabolism was highly controlled by pyrimidine metabolic rate-limiting enzymes [24]. CAD, CTPS, CTPS2, DHODH, DTYMK, NT5C2, NT5C3, RRM1, RRM2, TK1, TK2, TYMS, UCK2, and UCKL1 were reported pyrimidine metabolic rate-limiting enzymes [24]. The expression levels of those pyrimidine metabolic rate-limiting enzymes in lung normal and tumor tissues were investigated in GSE7670, GSE10072, GSE18842, GSE19188, GSE27262, GSE31908, GSE33532, and GSE75324 datasets. As illustrated in the heatmaps, pyrimidine metabolic rate-limiting enzymes CAD, CTPS, CTPS2, DHODH, DTYMK, NT5C3, RRM1, RRM2, TK2, TYMS, UCK2, and UCKL1 were upregulated in lung cancer tissues (Fig. 2). However, TK2 and NT5C2 were relatively downregulated in lung cancer tissues (Fig. 2).

Similar results were derived from TCGA LUAD dataset. Compared with the normal lung tissues, pyrimidine metabolic rate-limiting enzymes CAD, CTPS, CTPS2, DTYMK, NT5C3, RRM1, RRM2, TK2, TYMS, UCK2, and UCKL1 were all highly expressed in lung cancer tissues. However, TK2 and NT5C2 were downregulated in lung cancer tissues (Fig. 2). And there was no significant difference of $\beta$-actin (ACTB) expression levels in normal and lung cancer tissues (Fig. 2).

\section{The expression levels of purinergic receptors in lung cancer cells}

Purinergic receptors comprise two different sub-families, ionotropic P2X and metabotropic P2Y receptors [31, 32]. Next, we determined the expression levels of $\mathrm{P} 2 \mathrm{X}$ sub-families P2RX1-7 and P2Y receptors P2RY1, P2RY2, P2RY4, P2RY5 (LPAR6), P2RY6, P2RY7 (LTB4R), P2RY8, P2RY9 (LPAR4),
P2RY10-14 in normal lung tissues and lung cancer tissues. As depicted in GSE7670, GSE10072, GSE18842, GSE19188, GSE27262, GSE31908, GSE33532, GSE75324, and TCGA LUAD datasets, compared with the normal lung tissues, purinergic receptors P2RX1, P2RX7, P2RY12, P2RY13, and P2RY14 were relatively downregulated in lung cancer tissues (Fig. 3). However, the expression levels of other purinergic receptors in normal and lung cancer tissues were not significantly different (Fig. 3). Also, the expression levels of nucleoside transporter SLC28A3 were not changed in lung cancer tissues (Fig. 3).

\section{Pyrimidine metabolic rate-limiting enzymes are up- regulated in metastatic lung cancer cells and associ- ated with lung cancer recurrence}

LUAD cells can spread to the lymph nodes, adrenal glands, bones, and the brain [33]. PC9 BrM is a sub-population cells lines derived from parental PC9 LUAD cells, and with high brain metastasis [34]. We found that compared with parental PC9 cells, pyrimidine metabolic rate-limiting enzymes NT5C2, TK2, CAD, DTYMK, DHODH, RRM1, TK1, RRM2, TYMS, and CTPS were all highly expressed in PC9 BrM cells (Fig. 4a). Moreover, using MSKCC dataset, we showed that LUAD patients with high expression levels of CAD, RRM2, TK1, TYMS, or UCK2 were with high recurrence probability (Fig. 4b). However, purinergic receptors P2RX1, P2RX2, P2RY13, and P2RY14 were not associated with the tumor recurrence of lung cancer (Fig. 4c).

\section{Expression levels of pyrimidine metabolic rate- limiting enzymes are associated with the tumor overall survival in lung cancer: analysis from Gene Expression Omnibus datasets}

The Kaplan-Meier plotter is an online survival analysis tool to rapidly assess the prognostic effects of genes using the integrated GEO microarray data derived from 1926 lung cancer patients $[29,30]$. Using Kaplan-Meier plotter, the present study showed that high expression levels of pyrimidine metabolic rate-limiting enzymes CAD, CTPS, DHODH, DTYMK, RRM1, RRM2, TK1, TYMS, and UCK2 were unfavorable prognostic markers in patients with lung cancer (Fig. 5a). However, consistent with the decreased expression levels of NR5C2 and TK2 in lung cancer tissues, patients with higher expression levels of NR5C2 and TK2 had better prognosis than patients with low expression levels of those genes (Fig. 5a).

We also showed that contrast with the unfavorable prognosis of pyrimidine metabolic rate-limiting enzymes, purinergic receptors P2RX1, P2RX2, P2RX7, P2RY12, P2RY13, and $\mathrm{P} 2 \mathrm{RY} 14$ were favorable prognostic markers in patients with lung cancer (Fig. 5b). However, other purinergic receptors had no prognostic effects. 
a

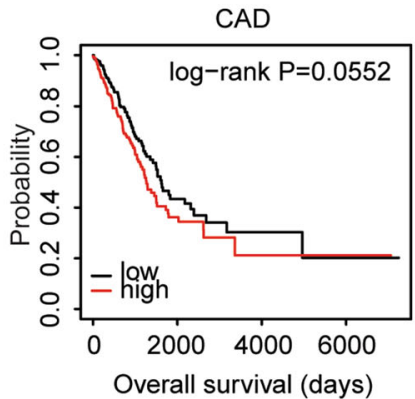

NT5C3
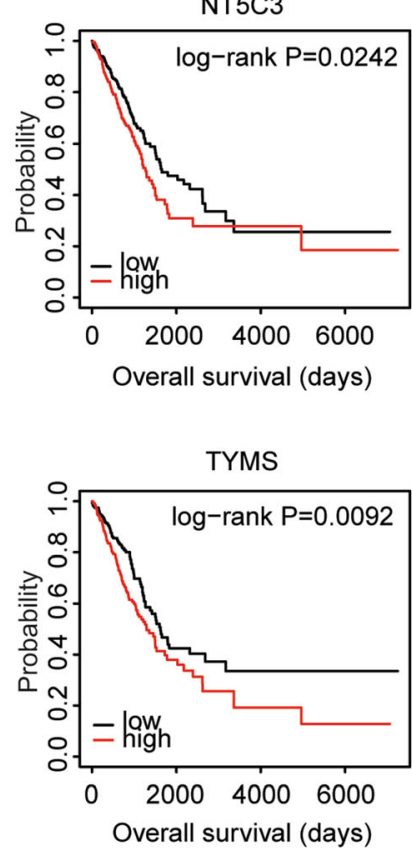

b

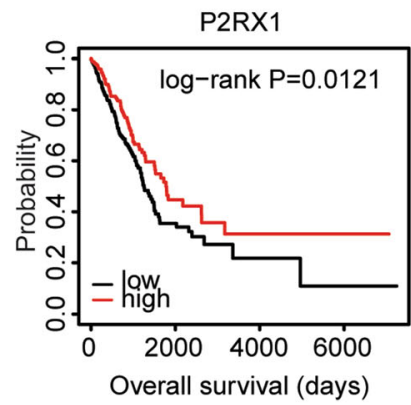

P2RY12

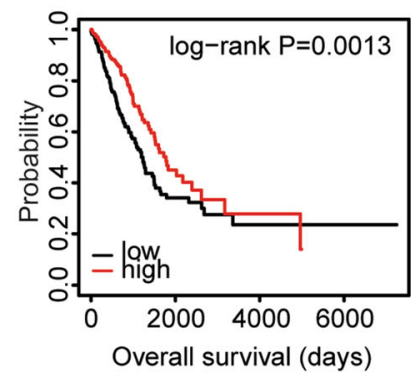

CTPS

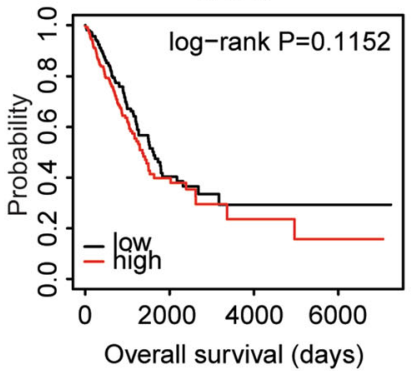

RRM1
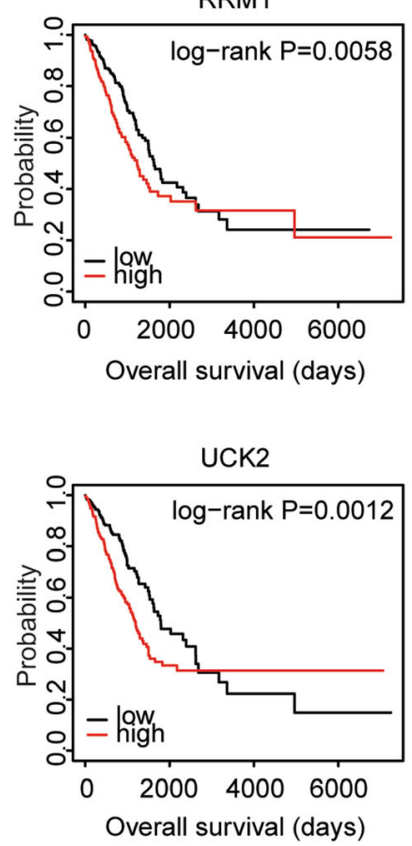
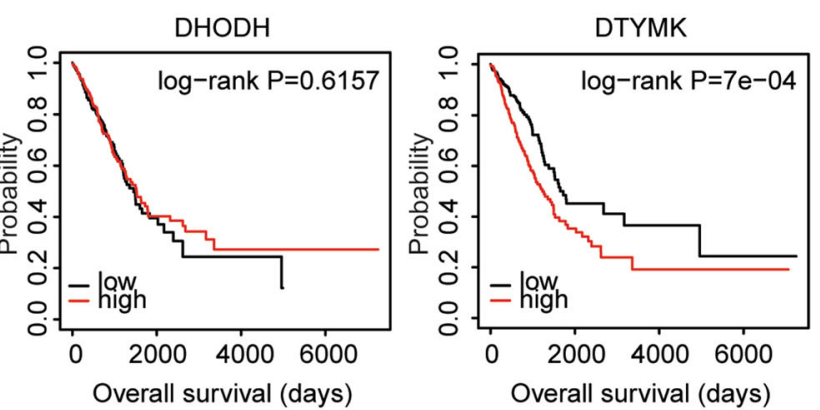

RRM2
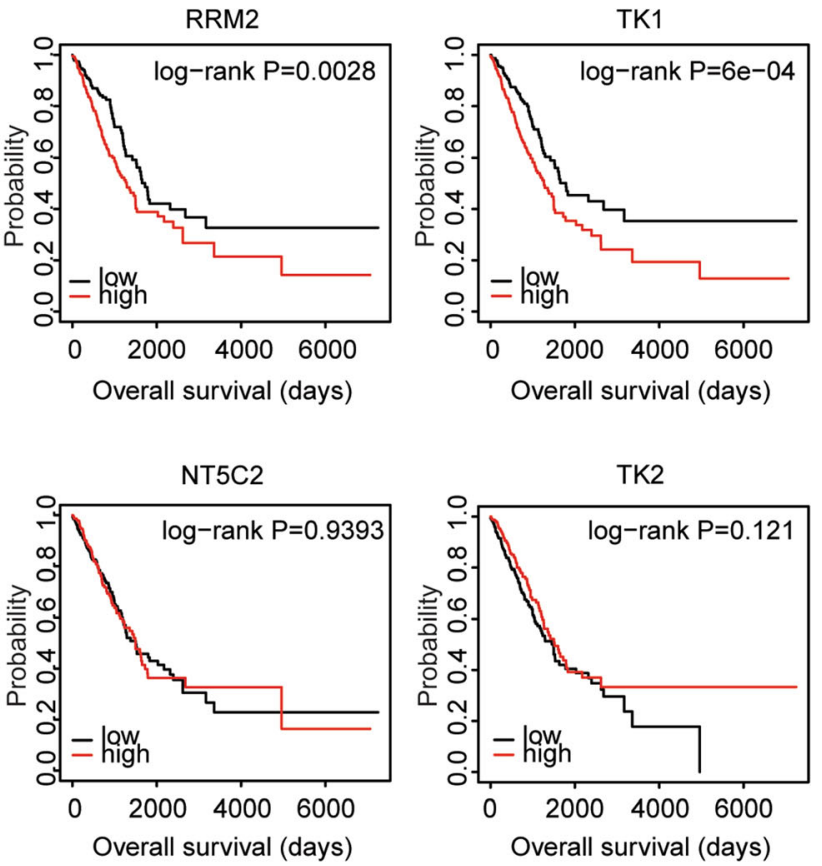
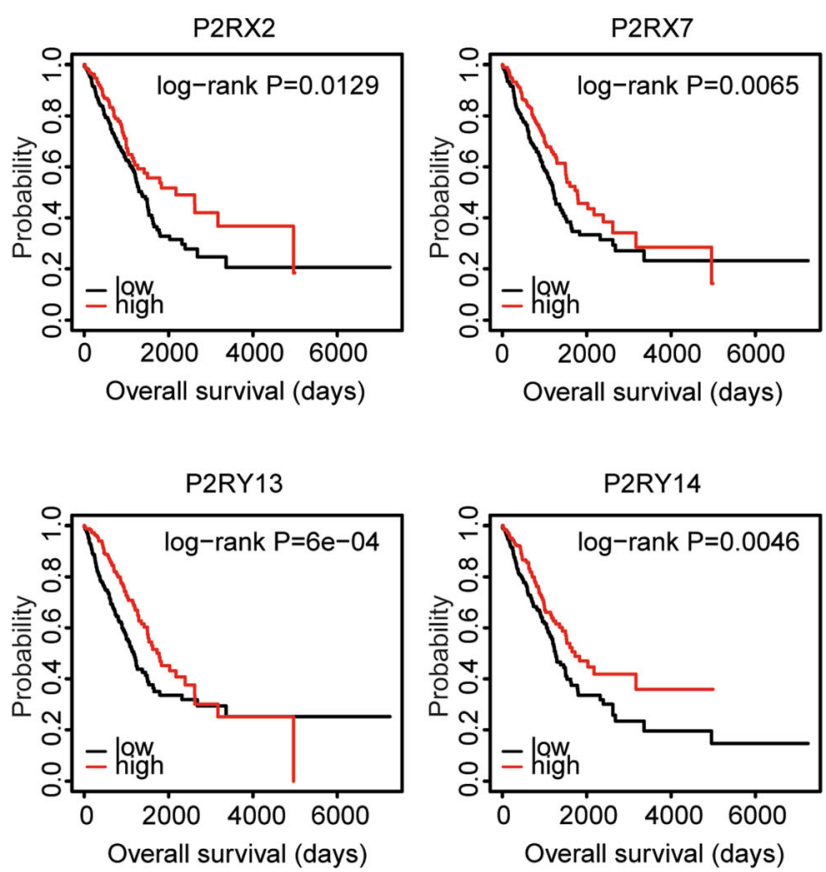
Fig. 6 Expression levels of pyrimidine metabolic rate-limiting enzymes are associated with the tumor overall survival in lung adenocarcinoma: analysis from TCGA LUAD dataset. a The Kaplan-Meier plotters demonstrated the associations between pyrimidine metabolic rate-limiting enzymes and overall survival in lung cancer using the TCGA LUAD dataset. The log-rank test was used to determine the overall survival $P$ value. b The Kaplan-Meier plotters demonstrated the prognostic effects of the expression levels of purinergic receptors P2RX1, P2RX2, P2RX7, P2RY12, P2RY13, and P2RY14 in lung cancer

\section{Expression levels of pyrimidine metabolic rate- limiting enzymes are associated with the tumor overall survival in lung adenocarcinoma: analysis from The Cancer Genome Atlas lung adenocarcinoma dataset}

Furthermore, using TCGA LUAD dataset, we confirmed the prognostic effects of pyrimidine metabolic rate-limiting enzymes and purinergic receptors. Similarly, the Kaplan-Meier survival analysis showed that pyrimidine metabolic ratelimiting enzymes DTYMK, NT5C3, RRM1, RRM2, TK1, TYMS, and UCK2 were all associated with adverse prognosis in the lung cancer (Fig. 6a). Patients with high expression levels of DTYMK, NT5C3, RRM1, RRM2, TK1, TYMS, or UCK2 were with low overall survival. However, we found that CAD, CTPS, DHODH, NR5C2, and TK2 had no prognostic effects in TCGA LUAD dataset (Fig. 6a). And only purinergic receptors P2RX1, P2RX2, P2RX7, P2RY12,
P2RY13, and P2RY14 were associated with good prognosis in the lung cancer (Fig. 6b).

The prognostic significance of pyrimidine metabolic rate-limiting enzymes in patients with LUSC was also tested using TCGA LUSC dataset. However, unlike LUAD, pyrimidine metabolic rate-limiting enzymes CAD, CTPS, DHODH, DTYMK, NT5C3, RRM1, RRM2, TK1, TYMS, UCK2, and NT5C2 had no prognostic effects in LUSC (Fig. S1). Only high expression levels of TK2 were associated with the adverse prognostic outcomes in LUSC (Fig. S1).

\section{Increased expression levels of the pyrimidine metabolic rate-limiting enzymes in lung cancer cells are induced by DNA hypomethylation}

Next, we tried to determine the mechanisms that induced the high expression levels of pyrimidine metabolic rate-limiting enzymes in lung cancer. The high expression levels of oncogenes are usually mediated by hypo-DNA methylation, DNA amplification, and gene mutation [35]. Using the DNA methylation data deposited in GSE32867 and GSE62948 datasets, we analyzed the DNA methylation intensity of the pyrimidine metabolic rate-limiting enzymes in normal lung tissues and lung cancer tissues.

Compared with the lung normal tissues, the pyrimidine metabolic rate-limiting enzymes CAD, RRM2, and TK1 were hypo-methylated in lung cancer tissues a

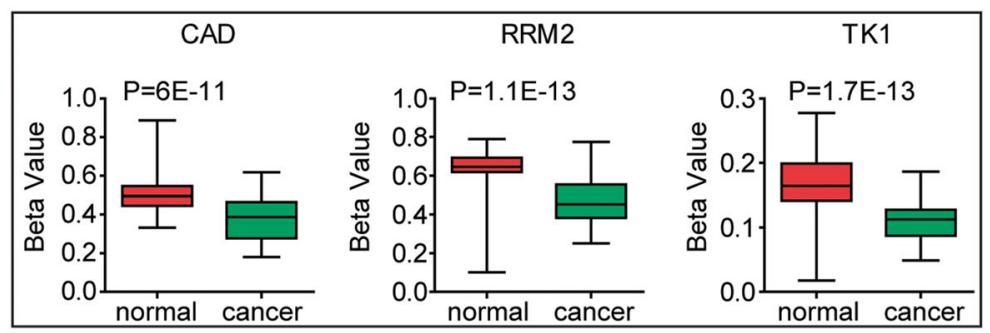

b

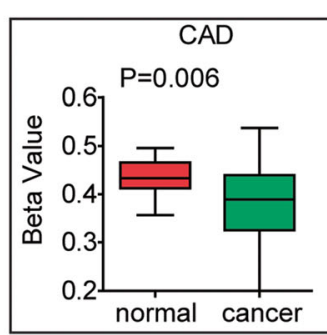

GSE62948
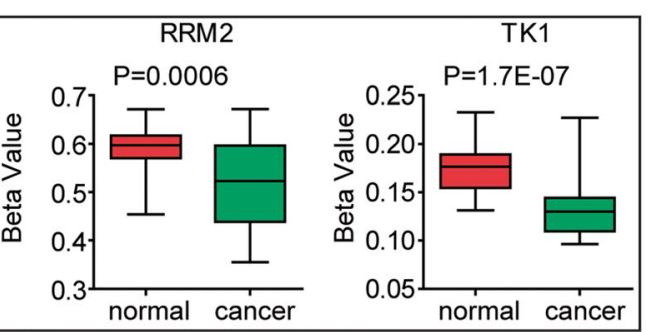

C

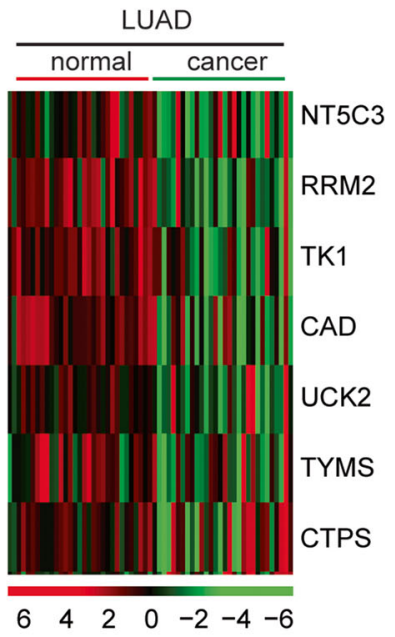

CAD, RRM2, and TK1 genes in normal lung tissues and lung adenocarcinoma tissues in GSE62948 dataset. $\mathbf{c}$ Heatmaps demonstrated the methylation level ( $\beta$ value) of the pyrimidine metabolic rate-limiting enzymes in normal and tumor tissues in LUAD. Hypermethylated (red), hypomethylated (green) and unchanged (black) genes were delineated
Fig. 7 Increased expression levels of the pyrimidine metabolic ratelimiting enzymes in lung cancer cells are induced by DNA hypomethylation. a Box plots demonstrated the DNA methylation intensity ( $\beta$ value) of pyrimidine metabolic rate limiting enzymes CAD, RRM2, and TK1 in normal lung tissues and lung adenocarcinoma tissues in GSE32867 dataset. b Box plots demonstrated the DNA methylation intensity of 
a

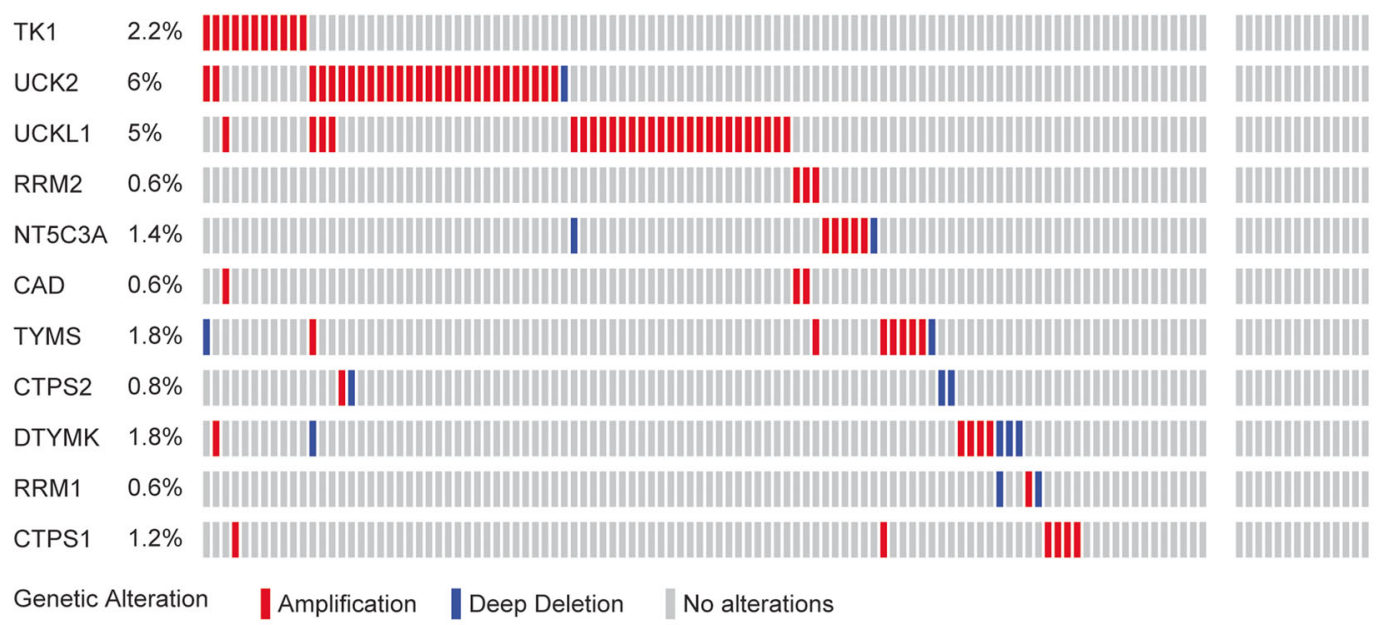

b

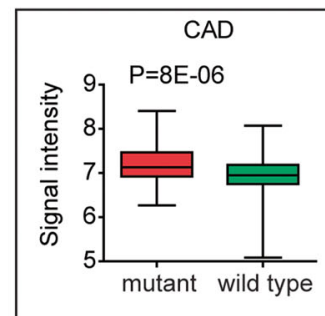

RRM1

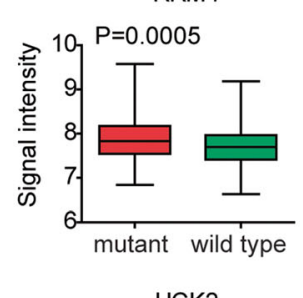

UCK2
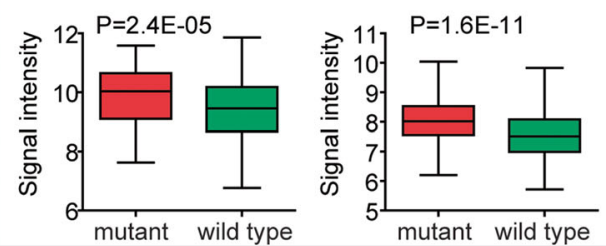

Fig. 8 Increased expression levels of the pyrimidine metabolic ratelimiting enzymes in lung cancer cells are induced by DNA amplification and TP53 mutation. a Oncoprints demonstrated the alteration frequency of pyrimidine metabolic rate-limiting enzymes in LUAD. Each line represented one patient. b Box plots demonstrated the expression levels of

derived from GSE32867 dataset (Fig. 7a). Similar results were obtained in GSE62948 dataset that the DNA methylation intensity of CAD, RRM2, and TK1 was lower in lung cancer tissues, compared with normal lung tissues (Fig. 7b). Also, in TCGA LUAD dataset, pyrimidine metabolic rate-limiting enzymes RRM2, TK1, CAD, UCK2, TYMS, and CTPS exhibited hypo-DNA methylation in LUAD tissues (Fig. 7c).
C

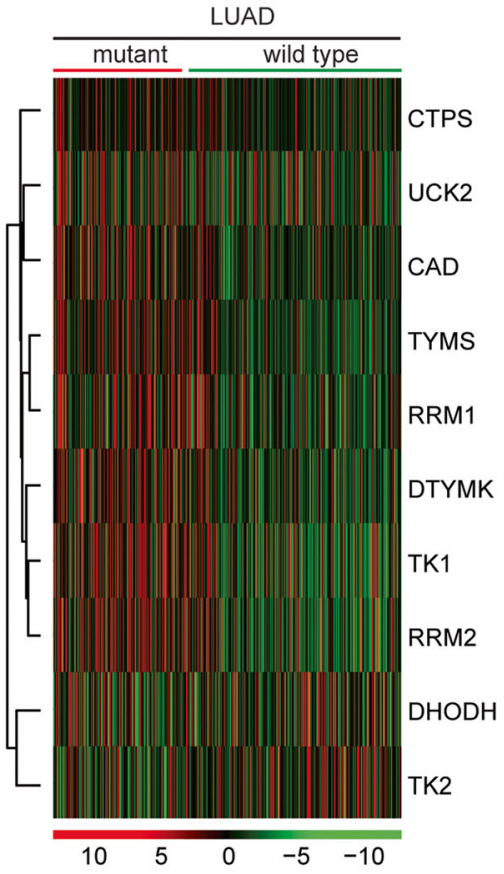

the pyrimidine metabolic rate limiting enzymes in patients with lung cancer. $P$ values indicated the differences between patients with TP53 mutant and TP53 wild-type. c Heatmap demonstrated the expression levels of the pyrimidine metabolic rate-limiting enzymes in TP53 mutant and TP53 wild-type LUAD patients

\section{Increased expression levels of the pyrimidine metabolic rate-limiting enzymes in lung cancer cells are induced by DNA amplification and TP53 mutation}

Another factor determining the activation of pyrimidine metabolic rate-limiting enzymes in lung cancer cells was genomic aberration, particularly DNA amplification. We showed that $6 \%$ lung cancer patients were with UCK2 amplification and 
Fig. 9 Pyrimidine metabolic rate limiting enzymes are highly correlated in lung cancer. a Corrplots demonstrated the correlation between pyrimidine metabolic rate-limiting enzymes in the GSE30219 and TCGA LUAD dataset. The color and the size of the circle represented the correlation coefficients. b

Multivariate Cox regression was used to test the relationships of pyrimidine metabolic ratelimiting enzyme expressions and overall survival in lung cancer patients in the GSE30219 and TCGA LUAD dataset a

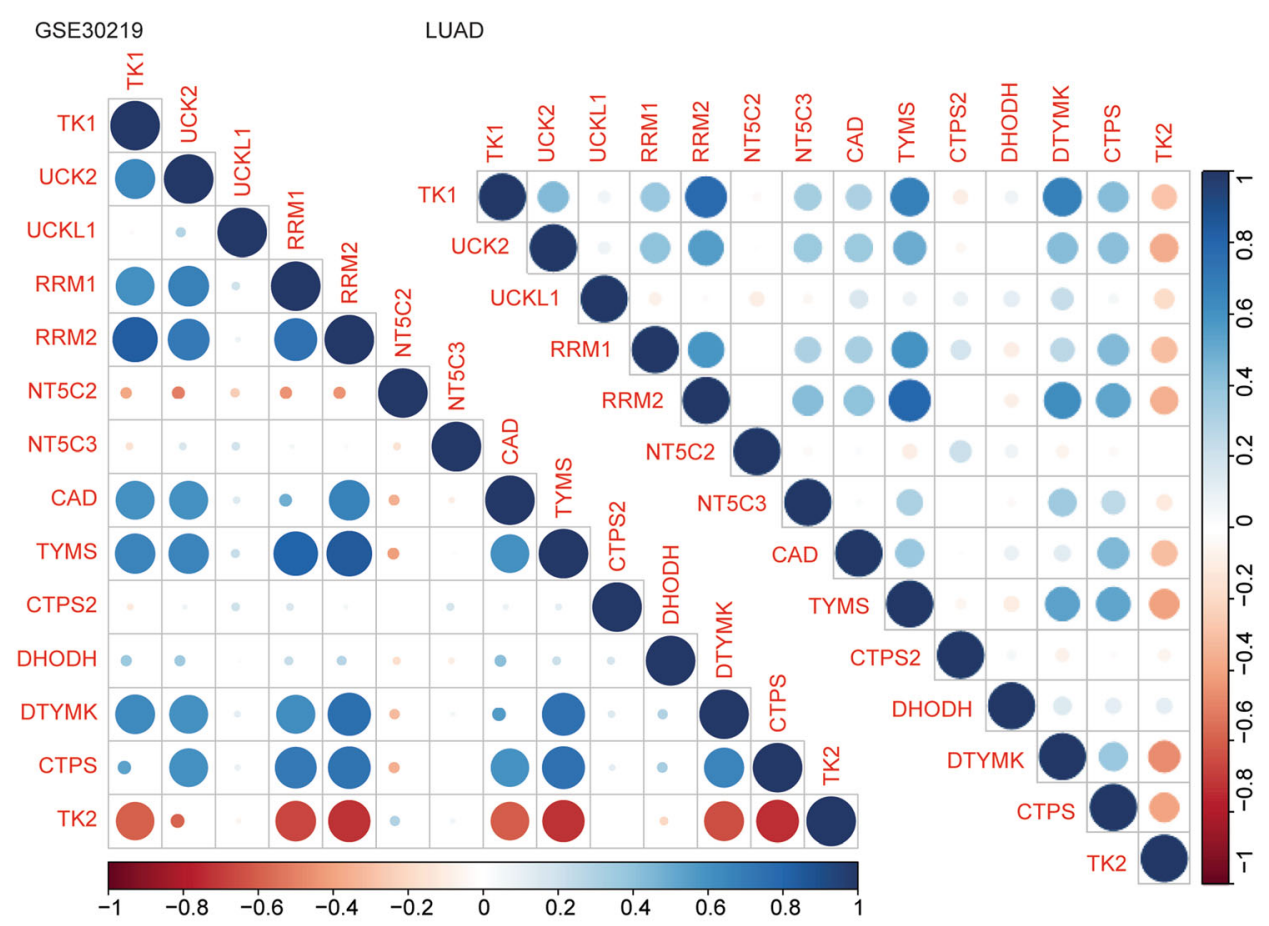

b

\begin{tabular}{lcccc}
\multicolumn{5}{c}{ GSE30219 } \\
\hline Variable & Coefficient & HR & p-value & $95 \% \mathrm{Cl}$ \\
\hline TK1 & -0.14 & 0.87 & 0.32 & $0.67-1.14$ \\
UCK2 & -0.06 & 0.94 & 0.65 & $0.76-1.18$ \\
UCKL1 & 0.005 & 1 & 0.99 & $0.55-1.84$ \\
RRM1 & 0.04 & 1.04 & 0.8 & $0.77-1.41$ \\
RRM2 & 0.42 & 1.52 & 0.0009 & $1.19-1.94$ \\
NT5C2 & -0.25 & 0.77 & 0.39 & $0.44-1.38$ \\
NT5C3 & 0.01 & 1.01 & 0.94 & $0.76-1.35$ \\
CAD & -0.17 & 0.84 & 0.6 & $0.45-1.58$ \\
TYMS & -0.043 & 0.96 & 0.79 & $0.7-1.31$ \\
CTPS2 & 0.013 & 1.01 & 0.93 & $0.77-1.33$ \\
DHODH & -0.08 & 0.92 & 0.75 & $0.58-1.48$ \\
DTYMK & 0.52 & 1.68 & 0.085 & $0.93-3.05$ \\
CTPS & -0.09 & 0.91 & 0.49 & $0.7-1.19$ \\
TK2 & -0.27 & 0.76 & 0.35 & $0.43-1.35$ \\
\hline & & & &
\end{tabular}

\begin{tabular}{lcccc}
\multicolumn{5}{c}{ LUAD } \\
\hline Variable & Coefficient & HR & p-value & $95 \% \mathrm{Cl}$ \\
\hline TK1 & 0.079 & 1.08 & 0.48 & $0.87-1.35$ \\
UCK2 & 0.08 & 1.08 & 0.28 & $0.94-1.25$ \\
UCKL1 & -0.32 & 0.73 & 0.02 & $0.56-0.95$ \\
RRM1 & -0.12 & 0.89 & 0.52 & $0.62-1.27$ \\
RRM2 & 0.04 & 1.04 & 0.75 & $0.81-1.34$ \\
NT5C2 & -0.06 & 0.94 & 0.7 & $0.7-1.27$ \\
NT5C3 & 0.14 & 1.15 & 0.27 & $0.89-1.49$ \\
CAD & 0.04 & 1.04 & 0.72 & $0.82-1.34$ \\
TYMS & 0.11 & 1.11 & 0.37 & $0.88-1.41$ \\
CTPS2 & 0.3 & 1.35 & 0.04 & $1-1.78$ \\
DHODH & -0.12 & 0.89 & 0.4 & $0.67-1.17$ \\
DTYMK & 0.16 & 1.17 & 0.36 & $0.83-1.65$ \\
CTPS & -0.06 & 0.94 & 0.68 & $0.72-1.24$ \\
TK2 & 0.007 & 1 & 0.95 & $0.77-1.32$ \\
\hline
\end{tabular}

5\% lung cancer patients were with UCKL1 amplification (Fig. 8a). Also, TK1 amplification occurred in 2.2\% lung cancer patients (Fig. 8a). However, other pyrimidine metabolic rate-limiting enzymes were without DNA amplification in lung cancer tissues (Fig. 8a).

TP53 is a critical regulator of multiple metabolism signaling pathways in lung cancer cells [36-38]. Loss of TP53 functions induces uncontrolled pyrimidine synthesis [39]. The present study assessed whether TP53 regulated the expression levels of the pyrimidine metabolic rate-limiting enzymes. We found that pyrimidine metabolic rate-limiting enzymes CAD,
CTPS, DTYMK, RRM1, RRM2, TYMS, UCK2, and TK1 were all highly expressed in TP53 mutant lung cancer patients (Fig. 8b). Interestingly, TK2 which was downregulated in lung tumor tissues was highly expressed in lung cancer patients with wild type TP53 (Fig. 8b).

Those results were further validated in the TCGA LUAD dataset. The expression levels of pyrimidine metabolic ratelimiting enzymes CAD, CTPS, DTYMK, RRM1, RRM2, TYMS, UCK2, and TK1 were particularly higher in TP53 mutant lung cancer patients (Fig. 8c). And the expression levels of TK2 were lower in TP53 mutant lung cancer patients 


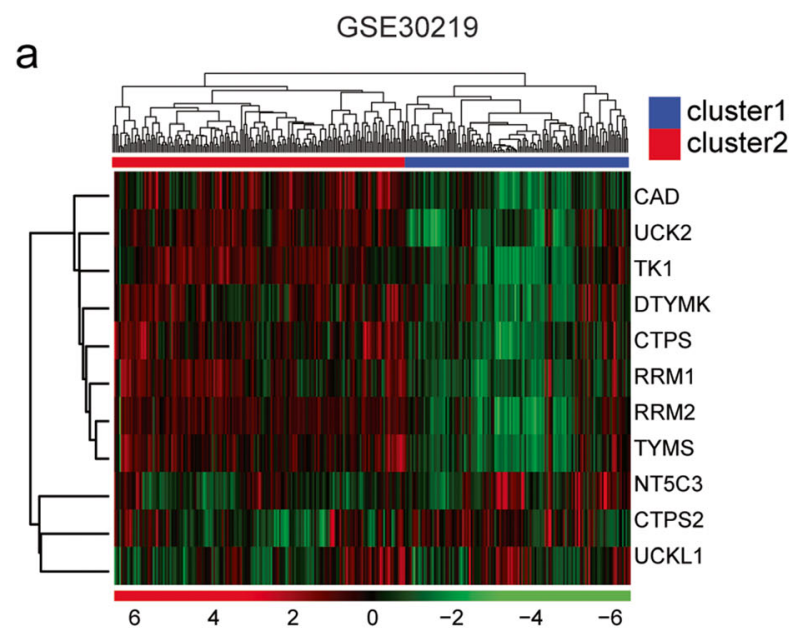

b
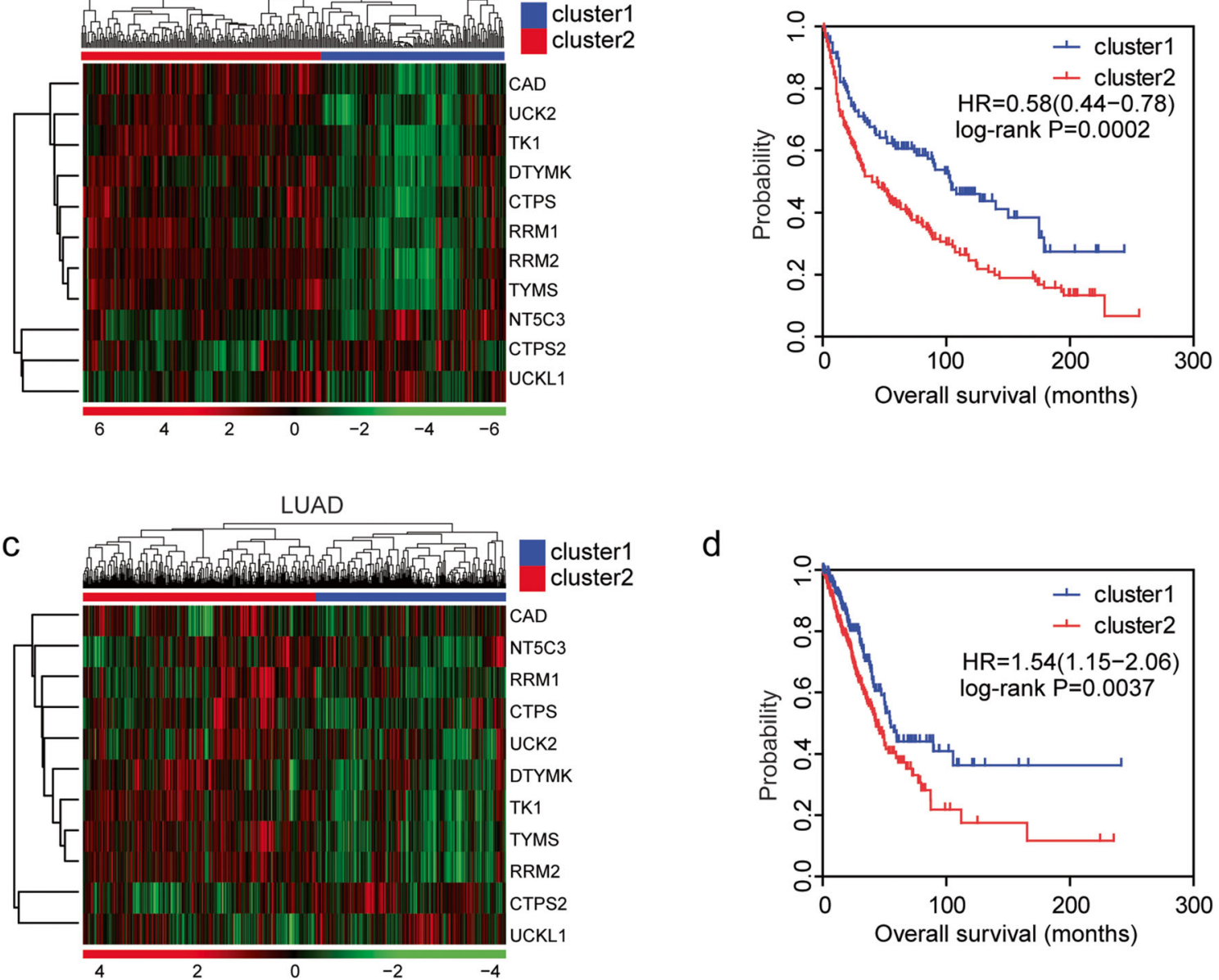

d

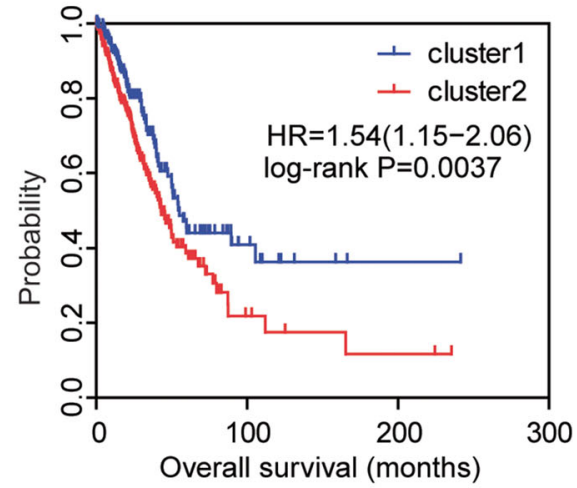

Fig. 10 Combined pyrimidine metabolic rate-limiting enzymes have significant prognostic effects of in lung cancer. a Unsupervised clustering heatmap showed the division of two clusters of lung cancer patients by the expression levels of pyrimidine metabolic rate-limiting enzymes in GSE30219 dataset. Each line represented one patient. b The KaplanMeier plotter demonstrated the different clinical outcomes of those two clusters of lung cancer patients in GSE30219 dataset. The log-rank test

(Fig. 8c). Overall, our results suggested that hypo-DNA methylation, DNA amplification, and TP53 mutation were combined contributing to the high expression levels of pyrimidine metabolic rate-limiting enzymes in lung cancer cells.

\section{Pyrimidine metabolic rate-limiting enzymes are highly correlated in lung cancer}

Using Spearman's correlation, we found the high correlations of pyrimidine metabolic rate-limiting enzymes. Particularly, RRM2 was highly associated with TK1, RRM1, TYMS, and DTYMK in GSE30219 dataset (Fig. 9a). However, NT5C2 and TK2 were negatively correlated with other pyrimidine metabolic rate-limiting enzymes (Fig. 9a). Similar results were obtained from TCGA LUAD dataset. RRM2 was positively correlated with other pyrimidine metabolic ratetering heatmap showed the division of two clusters of lung cancer patients by the expression levels of pyrimidine metabolic rate-limiting enzymes in TCGA LUAD dataset. d The Kaplan-Meier plotter demonstrated the different clinical outcomes of those two clusters of lung cancer patients in TCGA LUAD dataset. The log-rank test was used to determine the overall survival $P$ value

limiting enzymes, while TK2 was negatively correlated with other pyrimidine metabolic rate-limiting enzymes (Fig. 9a).

Furthermore, we used multivariate Cox regression analysis to determine the connections between the pyrimidine metabolic rate-limiting enzymes. It was revealed that RRM2 was an independent prognostic marker in lung cancer in GSE30219 dataset (Fig. 9b). In LUAD dataset, all pyrimidine metabolic ratelimiting enzymes were interconnected with each other and those genes were not independent prognostic markers (Fig. 9b).

\section{Combined pyrimidine metabolic rate-limiting en- zymes have significant prognostic effects of in lung cancer}

Next, we tested the combined prognostic effects of pyrimidine metabolic rate-limiting enzymes in lung 
cancer. Lung cancer patients were divided into two clusters based on the unsupervised clustering of the expression levels of pyrimidine metabolic rate-limiting enzymes in GSE30219 dataset (Fig. 10a). The cluster1 lung patients were with lower expression levels of CAD, CTPS, RRM1, RRM2, DTYMK, TK1, TYMS, and UCK2 (Fig. 10a). Lung cancer patients in cluster1 were with longer overall survival time, compared with lung cancer patients in cluster 2 (Fig. 10b).

Similarly, the patients were divided into two clusters by the unsupervised clustering of the pyrimidine metabolic ratelimiting enzymes in TCGA LUAD dataset (Fig. 10c). CAD, CTPS, RRM1, RRM2, DTYMK, TK1, TYMS, and UCK2 were downregulated in cluser1 lung cancer patients (Fig. 10c). Lung cancer patients in cluster1 demonstrated better prognostic outcomes, compared with lung cancer patients in cluster 2 (Fig. 10d).

\section{Pyrimidine metabolic rate-limiting enzymes are up- regulated in multiple types of tumor}

Comprehensively, using TCGA database, we investigated the expression levels of pyrimidine metabolic ratelimiting enzymes across different types of cancer. The expression levels of the pyrimidine metabolic ratelimiting enzymes in normal tissues and corresponding tumor tissues were investigated in bladder urothelial carcinoma (BLCA), breast invasive carcinoma (BRCA), colon adenocarcinoma (COAD), esophageal carcinoma (ESCA), kidney renal papillary cell carcinoma (KIRP), liver hepatocellular carcinoma (LIHC), lung squamous cell carcinoma (LUSC), stomach adenocarcinoma (STAD), and thyroid cancer (THCA) (Fig. 11). As illustrated in the heatmaps, pyrimidine metabolic ratelimiting enzymes CAD, CTPS, CTPS2, DHODH, DTYMK, NT5C3, RRM1, RRM2, TK2, TYMS, UCK2, and UCKL1 were highly expressed in tumor tissues (Fig. 11). However, TK2 and NT5C2 were not significantly upregulated in tumor tissues (Fig. 11). Moreover, $\beta$-actin was not altered in cancer tissues in most types of tumor (Fig. 11). These results indicated the universal importance of pyrimidine metabolic rate-limiting enzymes in the development of cancer.

Using GSEA assay, we found that pyrimidine metabolism signaling pathway was only significantly enriched in BRCA and THCA (Fig. S2). Although, pyrimidine metabolic rate-limiting enzymes were upregulated in BLCA, COAD, ESCA, LIHC, and STAD, the pyrimidine metabolism signaling pathway was not significantly enriched (Fig. S2).
The association between the expression levels of pyrimidine metabolic rate-limiting enzymes and the tumor overall survival in liver cancer, breast cancer, or stomach cancer: analysis from breast invasive carcinoma, stomach adenocarcinoma, and liver hepatocellular carcinoma datasets

Like LUAD, pyrimidine metabolic rate-limiting enzymes were highly expressed in BRCA, LIHC, and STAD. However, in TCGA BRCA dataset, pyrimidine metabolic rate-limiting enzymes CAD, CTPS, DHODH, DTYMK, NT5C3, RRM1, RRM2, TK1, TYMS, UCK2, NT5C2, or TK2 demonstrated no prognostic effect (Fig. S3). Similarly, expression levels of pyrimidine metabolic rate-limiting enzymes had no clinical relevance in stomach cancer (Fig. S4). Only, TK1 was associated with better clinical outcomes (Fig. S4).

On the contrary, high expression levels of pyrimidine metabolic rate-limiting enzymes CAD, DTYMK, NT5C3, RRM1, RRM2, TK1, TYMS, and UCK2 were all associated with worse clinical outcomes in TCGA LIHC dataset (Fig. 12). Moreover, patients with higher expression levels of TK2 had better prognosis than patients with low expression levels of TK2 (Fig. 12). Those results highlighted the different prognostic effects of pyrimidine metabolic rate-limiting enzymes in different tumor types.

\section{Discussion}

Metabolic reprogramming is a hallmark of cancer [20] and provides critical information for cancer classification and clinical prognosis [40]. Here, we identified the clinical relevance of pyrimidine metabolic rate-limiting enzymes in lung cancer based on their mRNA expression patterns using GEO and TCGA datasets. Ideally, we should use the metabolic activities to determine the prognostic effects of the pyrimidine metabolic rate-limiting enzymes. However, previous results suggested that the metabolic reprogramming was caused by gene expression changes [41] and the expression profiles of metabolic pathway genes reflected the actual metabolic activities [42]. So, the expression patterns of metabolic genes had potential implications for clinical prognosis.

Lung cancer is a heterogeneous disease, including many different subtypes with different genetic and epigenetic abnormality [6]. Because of the complexity of lung cancer [43], results derived from GEO and TCGA datasets were not always consistent with each other. Also, the difference in treatment protocol and microarray platform in individual study limited the further applications of these findings [44]. For example, CAD, CTPS, and 

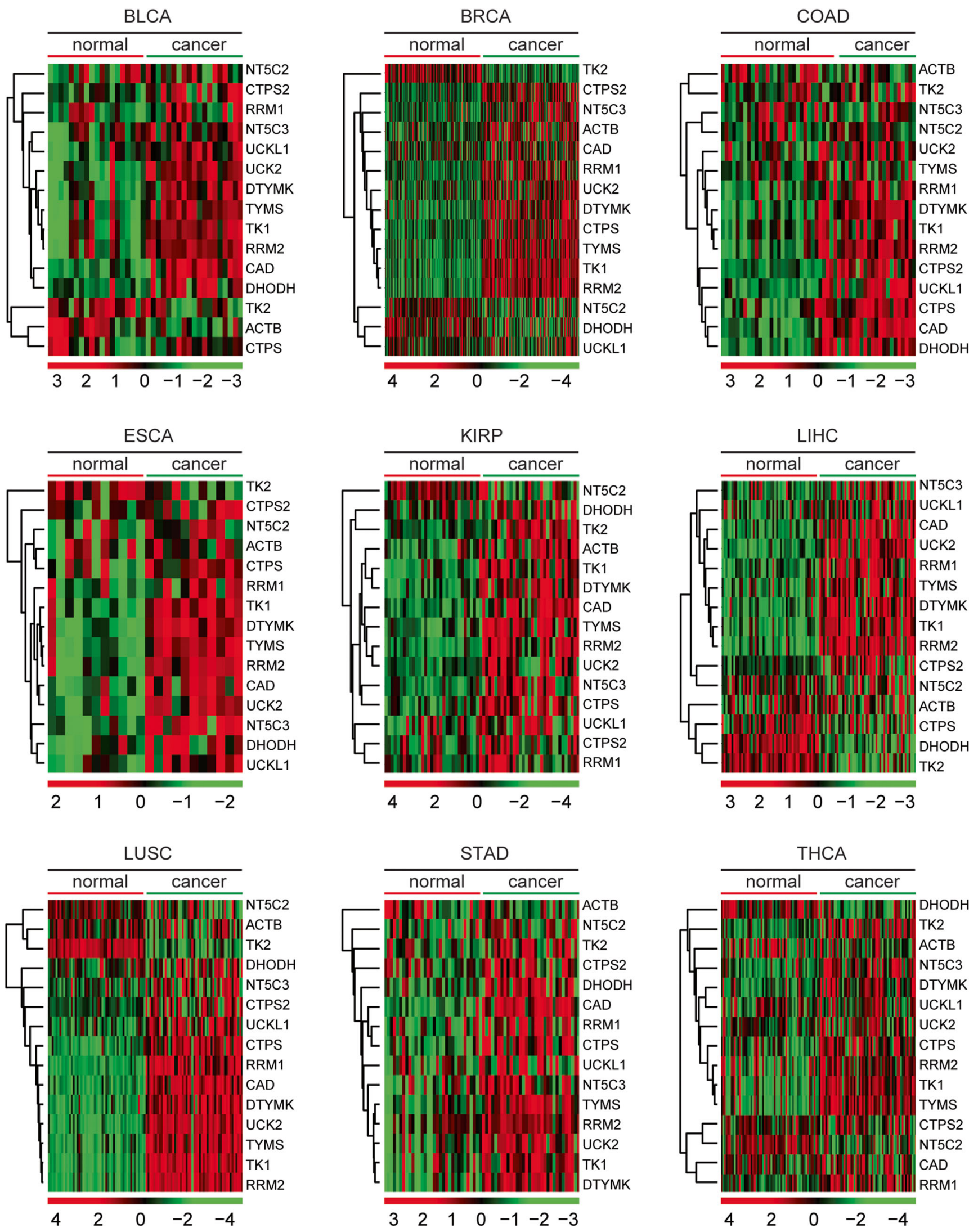
Fig. 11 Pyrimidine metabolic rate limiting enzymes are up-regulated in multiple types of tumor. Heatmaps demonstrated the expression levels ( $\log 2$ count) of pyrimidine metabolic rate limiting enzymes in normal and tumor samples in BLCA, BRCA, COAD, ESCA, KIRP, LIHC, LUSC, STAD, and THCA. Upregulated (red), downregulated (green), and unchanged (black) genes were delineated. BLCA, bladder urothelial carcinoma; BRCA, breast invasive carcinoma; COAD, colon adenocarcinoma; ESCA, esophageal carcinoma; KIRP, kidney renal papillary cell carcinoma; LIHC, liver hepatocellular carcinoma; LUSC, lung squamous cell carcinoma; STAD, stomach adenocarcinoma; THCA, thyroid cancer

DHODH had no prognostic effect in LUAD dataset but associated with the clinical outcomes in lung cancer patients derived from GEO datasets. To address this problem, we collected and studied multiple lung cancer GEO datasets and TCGA datasets. Our results suggested that the converged pyrimidine metabolism signaling pathway was generally altered in many datasets. And the pyrimidine metabolic rate-limiting enzymes DTYMK, NT5C3, RRM1, RRM2, TK1, TYMS, and UCK2 had particular values in lung cancer prognosis. Those highly expressed pyrimidine metabolic rate-limiting enzyme increased pyrimidine metabolism, facilitated the uncontrolled cell proliferation, and changed of the immune cell responses.

Some of the pyrimidine metabolic rate-limiting enzymes, such as TK1 [45], UCK2 [46], and RRM1 [47], were reported to be associated with the poor outcomes of lung cancer in systematic review or meta-analysis. Functional studies identified pyrimidine metabolic rate-limiting enzymes DHODH [48] and DTYMK [49] as therapeutic targets in lung cancer. Using the GEO and TCGA datasets, we confirmed the prognostic significance of pyrimidine metabolic rate-limiting enzymes TK1,

Prognostic significance of pyrimidine metabolic rate limiting enzymes in patients with liver cancer: analysis from TCGA LIHC dataset
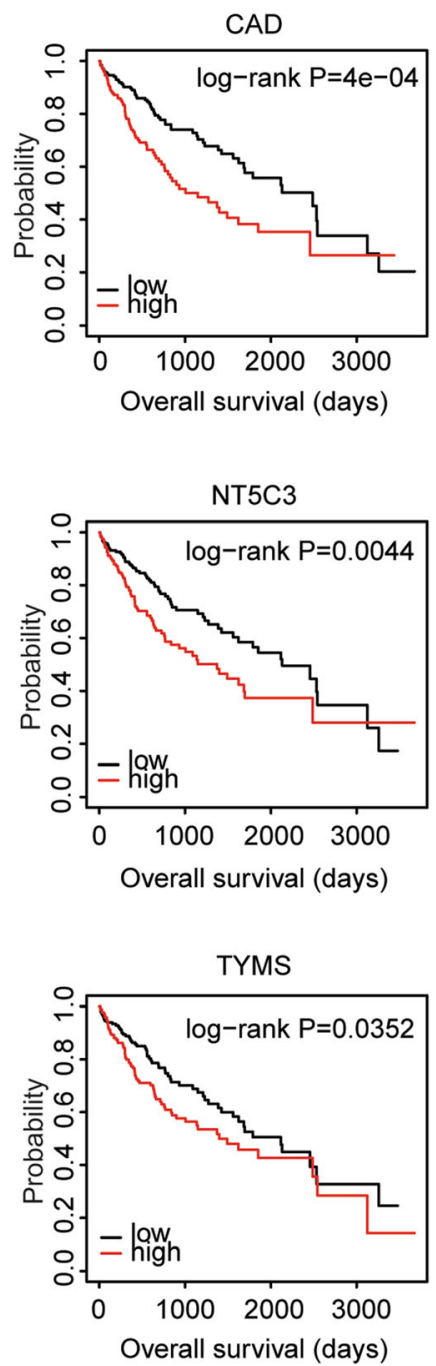

CTPS

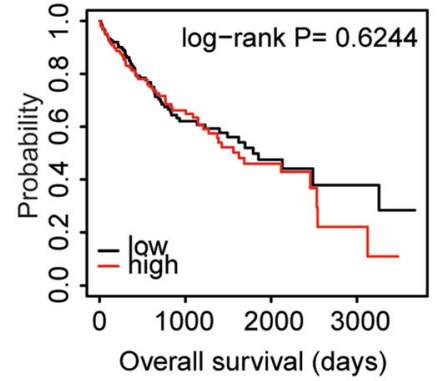

RRM1
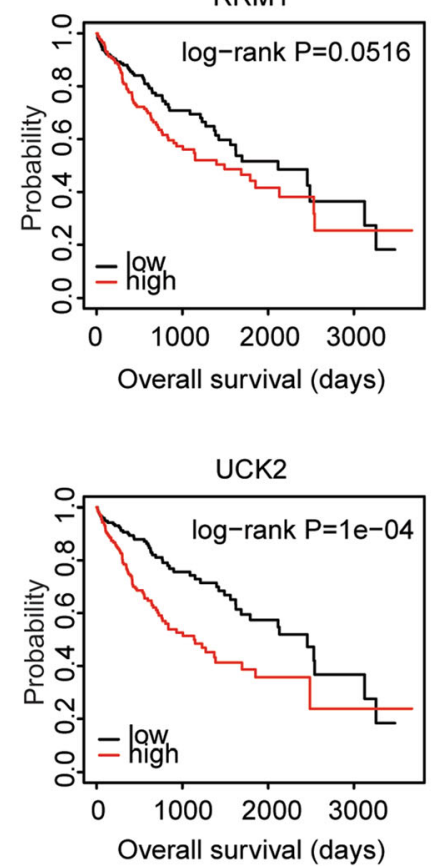

Fig. 12 Expression levels of pyrimidine metabolic rate-limiting enzymes are associated with the overall survival in liver cancer: analysis from TCGA LIHC dataset. The Kaplan-Meier plotters demonstrated the associations between pyrimidine metabolic rate limiting enzymes and overall
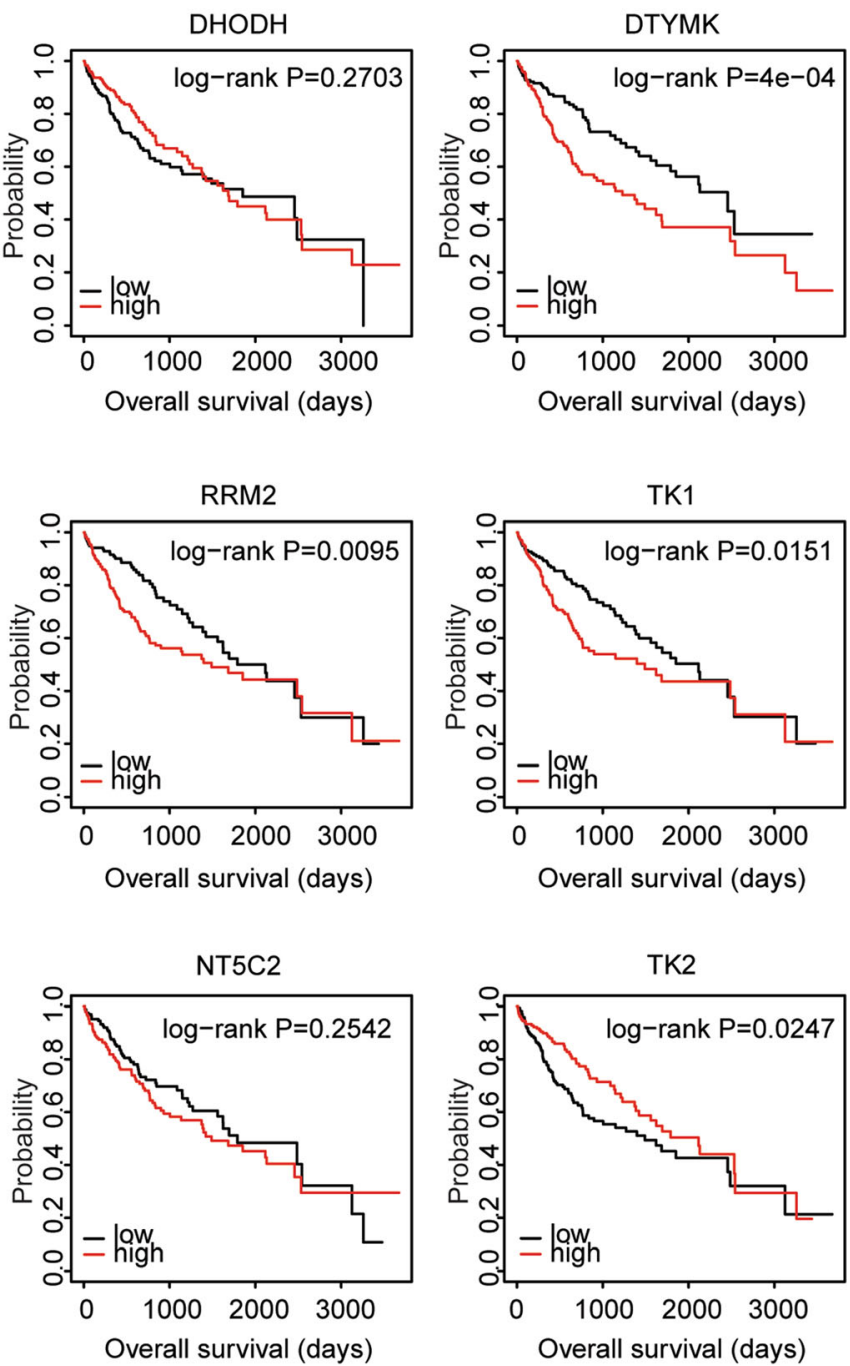

survival in liver cancer using the TCGA LIHC dataset. The log-rank test was used to determine the overall survival $P$ value. LIHC: liver hepatocellular carcinoma 
UCK2, and RRM1 in LUAD. Furthermore, we found that pyrimidine metabolic rate-limiting enzymes CAD, RRM2, DTYMK, TYMS, TK2, and NR5C2 were all associated with the clinical outcomes of lung cancer and liver cancer. However, the expression levels and prognostic effects of purinergic receptors in lung cancer were complex. It was reported that P2RX7 increased cancer invasiveness and metastasis and was adverse prognostic factor $[50,51]$. However, in our data, we found that P2RX7 was downregulated and was a good prognostic factor in lung cancer. Moreover, purinergic receptors P2RX1, P2RY12, P2RY13, and P2RY14 shared similar expression profiling and prognostic relevance. Those results suggested the complex functions of purinergic receptors in cancer development and should be further studied. Particularly, the purinergic receptors may influence the tumor immune cell responses by altering the tumor microenvironment.

The present study provided potential biomarkers for clinical prognosis of lung cancer. However, there were some limitations in this study. First, although the expression profiles of metabolic genes reflected the actual metabolic activities, the enzymatic activities of pyrimidine metabolic rate-limiting enzymes should be further tested. Second, clinical validations and functional studies were needed to reveal the inner mechanisms of how pyrimidine metabolic rate-limiting enzymes correlated with the clinical outcomes of lung cancer patients. Our results also suggested the different prognostic effects of pyrimidine metabolic rate-limiting enzymes in LUAD and LUSC. So, the clinical relevance the pyrimidine metabolic rate-limiting enzymes in different subtypes of lung cancer should also be further illustrated. In our further studies, we will address those limitations and provide a more precise and reliable prognostic signature based on the metabolic activities of the pyrimidine metabolic rate-limiting enzymes.

Authors' contributions HW.W and XR.W designed and performed data analysis. LP.X helped with the data analysis. HW.W wrote the manuscript. JZ and $\mathrm{HC}$ reviewed the manuscript and supervised the work. All the authors read and approved the final manuscript.

Funding information The present study was supported by grants from the Fujian Maternity and Child Health Hospital (grant nos. YCXB 18-10 and YCXM 19-04).

Data availability The datasets generated and/or analyzed during the current study are available in TCGA (tcga.xenahubs.net) and GEO (www. ncbi.nlm.nih.gov/geo) repositories.

\section{Compliance with ethical standards}

Conflict of interest The authors declare that they have no conflicts of interest.

Ethics approval and consent to participate Not applicable.

Consent for publication Not applicable.
Open Access This article is licensed under a Creative Commons Attribution 4.0 International License, which permits use, sharing, adaptation, distribution and reproduction in any medium or format, as long as you give appropriate credit to the original author(s) and the source, provide a link to the Creative Commons licence, and indicate if changes were made. The images or other third party material in this article are included in the article's Creative Commons licence, unless indicated otherwise in a credit line to the material. If material is not included in the article's Creative Commons licence and your intended use is not permitted by statutory regulation or exceeds the permitted use, you will need to obtain permission directly from the copyright holder. To view a copy of this licence, visit http://creativecommons.org/licenses/by/4.0/.

\section{References}

1. Siegel RL, Miller KD, Jemal A (2018) Cancer statistics, 2018. CA Cancer J Clin 68(1):7-30. https://doi.org/10.3322/caac.21442

2. Bray F, Ferlay J, Soerjomataram I, Siegel RL, Torre LA, Jemal A (2018) Global cancer statistics 2018: GLOBOCAN estimates of incidence and mortality worldwide for 36 cancers in 185 countries. CA Cancer J Clin 68(6):394-424. https://doi.org/10.3322/caac. 21492

3. Ferlay J, Colombet M, Soerjomataram I, Mathers C, Parkin DM, Pineros $\mathrm{M}$ et al (2019) Estimating the global cancer incidence and mortality in 2018: GLOBOCAN sources and methods. Int J Cancer 144(8):1941-1953. https://doi.org/10.1002/ijc.31937

4. Miller VA, Hirsh V, Cadranel J, Chen YM, Park K, Kim SW, Zhou C, Su WC, Wang M, Sun Y, Heo DS, Crino L, Tan EH, Chao TY, Shahidi M, Cong XJ, Lorence RM, Yang JCH (2012) Afatinib versus placebo for patients with advanced, metastatic non-smallcell lung cancer after failure of erlotinib, gefitinib, or both, and one or two lines of chemotherapy (LUX-lung 1): a phase $2 b / 3$ randomised trial. Lancet Oncol 13(5):528-538. https://doi.org/10. 1016/S1470-2045(12)70087-6

5. Moll HP, Pranz K, Musteanu M, Grabner B, Hruschka N, Mohrherr $\mathrm{J}$ et al (2018) Afatinib restrains K-RAS-driven lung tumorigenesis. Sci Transl Med 10(446). https://doi.org/10.1126/scitranslmed. aao2301

6. Herbst RS, Heymach JV, Lippman SM (2008) Lung cancer. N Engl J Med 359(13):1367-1380. https://doi.org/10.1056/ NEJMra0802714

7. Travis WD (2011) Pathology of lung cancer. Clin Chest Med 32(4): 669-692. https://doi.org/10.1016/j.ccm.2011.08.005

8. Cancer Genome Atlas Research N (2014) Comprehensive molecular profiling of lung adenocarcinoma. Nature. 511(7511):543-550. https://doi.org/10.1038/nature13385

9. Cancer Genome Atlas Research N (2012) Comprehensive genomic characterization of squamous cell lung cancers. Nature. 489(7417): 519-525. https://doi.org/10.1038/nature11404

10. Wu K, Zhang X, Li F, Xiao D, Hou Y, Zhu S, Liu D, Ye X, Ye M, Yang J, Shao L, Pan H, Lu N, Yu Y, Liu L, Li J, Huang L, Tang H, Deng Q, Zheng Y, Peng L, Liu G, Gu X, He P, Gu Y, Lin W, He H, Xie G, Liang H, An N, Wang H, Teixeira M, Vieira J, Liang W, Zhao X, Peng Z, Mu F, Zhang X, Xu X, Yang H, Kristiansen K, Wang J, Zhong N, Wang J, Pan-Hammarström Q, He J (2015) Frequent alterations in cytoskeleton remodelling genes in primary and metastatic lung adenocarcinomas. Nat Commun 6:10131. https://doi.org/10.1038/ncomms10131

11. Jiang J, Gu Y, Liu J, Wu R, Fu L, Zhao J, Guan Y (2016) Coexistence of p16/CDKN2A homozygous deletions and activating EGFR mutations in lung adenocarcinoma patients signifies a 
poor response to EGFR-TKIs. Lung Cancer 102:101-107. https:// doi.org/10.1016/j.lungcan.2016.10.015

12. Wang Z, Wang Z, Niu X, Liu J, Wang Z, Chen L, Qin B (2019) Identification of seven-gene signature for prediction of lung squamous cell carcinoma. Onco Targets Ther 12:5979-5988. https://doi. org/10.2147/OTT.S198998

13. Li Y, Gu J, Xu F, Zhu Q, Ge D, Lu C (2018) Transcriptomic and functional network features of lung squamous cell carcinoma through integrative analysis of GEO and TCGA data. Sci Rep 8(1):15834. https://doi.org/10.1038/s41598-018-34160-w

14. Li X, Shi Y, Yin Z, Xue X, Zhou B (2014) An eight-miRNA signature as a potential biomarker for predicting survival in lung adenocarcinoma. J Transl Med 12:159. https://doi.org/10.1186/ 1479-5876-12-159

15. Siriwardhana C, Khadka VS, Chen JJ, Deng Y (2019) Development of a miRNA-seq based prognostic signature in lung adenocarcinoma. BMC Cancer 19(1):34. https://doi.org/10.1186/ s12885-018-5206-8

16. Sui J, Yang S, Liu T, Wu W, Xu S, Yin L, Pu Y, Zhang X, Zhang Y, Shen B, Liang G (2019) Molecular characterization of lung adenocarcinoma: a potential four-long noncoding RNA prognostic signature. J Cell Biochem 120(1):705-714. https://doi.org/10.1002/ jcb. 27428

17. Lin T, Fu Y, Zhang X, Gu J, Ma X, Miao R et al (2018) A sevenlong noncoding RNA signature predicts overall survival for patients with early stage non-small cell lung cancer. Aging (Albany NY) 10(9):2356-2366. https://doi.org/10.18632/aging.101550

18. Song Q, Shang J, Yang Z, Zhang L, Zhang C, Chen J, Wu X (2019) Identification of an immune signature predicting prognosis risk of patients in lung adenocarcinoma. J Transl Med 17(1):70. https://doi. org/10.1186/s12967-019-1824-4

19. Yue C, Ma H, Zhou Y (2019) Identification of prognostic gene signature associated with microenvironment of lung adenocarcinoma. PeerJ. 7:e8128. https://doi.org/10.7717/peerj.8128

20. Hanahan D, Weinberg RA (2011) Hallmarks of cancer: the next generation. Cell. 144(5):646-674. https://doi.org/10.1016/j.cell. 2011.02.013

21. Shukla SK, Purohit V, Mehla K, Gunda V, Chaika NV, Vernucci E, King RJ, Abrego J, Goode GD, Dasgupta A, Illies AL, Gebregiworgis T, Dai B, Augustine JJ, Murthy D, Attri KS, Mashadova O, Grandgenett PM, Powers R, Ly QP, Lazenby AJ, Grem JL, Yu F, Matés JM, Asara JM, Kim JW, Hankins JH, Weekes C, Hollingsworth MA, Serkova NJ, Sasson AR, Fleming JB, Oliveto JM, Lyssiotis CA, Cantley LC, Berim L, Singh PK (2017) MUC1 and HIF-1alpha signaling crosstalk induces anabolic glucose metabolism to impart gemcitabine resistance to pancreatic cancer. Cancer Cell 32(1):71-87 e7. https://doi.org/10.1016/j.ccell. 2017.06.004

22. Zhang L, Zhang Z, Yu Z (2019) Identification of a novel glycolysisrelated gene signature for predicting metastasis and survival in patients with lung adenocarcinoma. J Transl Med 17(1):423. https:// doi.org/10.1186/s12967-019-02173-2

23. Rabinovich S, Adler L, Yizhak K, Sarver A, Silberman A, Agron S, Stettner N, Sun Q, Brandis A, Helbling D, Korman S, Itzkovitz S, Dimmock D, Ulitsky I, Nagamani SCS, Ruppin E, Erez A (2015) Diversion of aspartate in ASS1-deficient tumours fosters de novo pyrimidine synthesis. Nature. 527(7578):379-383. https://doi.org/ 10.1038/nature15529

24. Yeh HW, Lee SS, Chang CY, Hu CM, Jou YS (2017) Pyrimidine metabolic rate limiting enzymes in poorly-differentiated hepatocellular carcinoma are signature genes of cancer stemness and associated with poor prognosis. Oncotarget 8(44):77734-77751. https:// doi.org/10.18632/oncotarget.20774

25. Wang X, Yang K, Wu Q, Kim LJY, Morton AR, Gimple RC et al (2019) Targeting pyrimidine synthesis accentuates molecular therapy response in glioblastoma stem cells. Sci Transl Med 11(504). https://doi.org/10.1126/scitranslmed.aau4972

26. Brown KK, Spinelli JB, Asara JM, Toker A (2017) Adaptive reprogramming of de novo pyrimidine synthesis is a metabolic vulnerability in triple-negative breast cancer. Cancer Discov 7(4): 391-399. https://doi.org/10.1158/2159-8290.CD-16-0611

27. Chitale D, Gong Y, Taylor BS, Broderick S, Brennan C, Somwar R, Golas B, Wang L, Motoi N, Szoke J, Reinersman JM, Major J, Sander C, Seshan VE, Zakowski MF, Rusch V, Pao W, Gerald W, Ladanyi M (2009) An integrated genomic analysis of lung cancer reveals loss of DUSP4 in EGFR-mutant tumors. Oncogene. 28(31): 2773-2783. https://doi.org/10.1038/onc.2009.135

28. Subramanian A, Tamayo P, Mootha VK, Mukherjee S, Ebert BL, Gillette MA, Paulovich A, Pomeroy SL, Golub TR, Lander ES, Mesirov JP (2005) Gene set enrichment analysis: a knowledgebased approach for interpreting genome-wide expression profiles. Proc Natl Acad Sci U S A 102(43):15545-15550. https://doi.org/ 10.1073/pnas.0506580102

29. Gyorffy B, Surowiak P, Budczies J, Lanczky A (2013) Online survival analysis software to assess the prognostic value of biomarkers using transcriptomic data in non-small-cell lung cancer. PLoS One 8(12):e82241. https://doi.org/10.1371/journal.pone.0082241

30. Nagy A, Lanczky A, Menyhart O, Gyorffy B (2018) Validation of miRNA prognostic power in hepatocellular carcinoma using expression data of independent datasets. Sci Rep 8(1):9227. https:// doi.org/10.1038/s41598-018-27521-y

31. Di Virgilio F (2012) Purines, purinergic receptors, and cancer. Cancer Res 72(21):5441-5447. https://doi.org/10.1158/00085472.CAN-12-1600

32. Burnstock G, Di Virgilio F (2013) Purinergic signalling and cancer. Purinergic Signal 9(4):491-540. https://doi.org/10.1007/s11302013-9372-5

33. Hess KR, Varadhachary GR, Taylor SH, Wei W, Raber MN, Lenzi $\mathrm{R}$, Abbruzzese JL (2006) Metastatic patterns in adenocarcinoma. Cancer. 106(7):1624-1633. https://doi.org/10.1002/cncr.21778

34. Nguyen DX, Chiang AC, Zhang XH, Kim JY, Kris MG, Ladanyi $M$ et al (2009) WNT/TCF signaling through LEF1 and HOXB9 mediates lung adenocarcinoma metastasis. Cell. 138(1):51-62. https://doi.org/10.1016/j.cell.2009.04.030

35. Sun W, Bunn P, Jin C, Little P, Zhabotynsky V, Perou CM, Hayes DN, Chen M, Lin DY (2018) The association between copy number aberration, DNA methylation and gene expression in tumor samples. Nucleic Acids Res 46(6):3009-3018. https://doi.org/10. 1093/nar/gky131

36. Vousden KH, Ryan KM (2009) p53 and metabolism. Nat Rev Cancer 9(10):691-700. https://doi.org/10.1038/nrc2715

37. Gottlieb E, Vousden KH (2010) p53 regulation of metabolic pathways. Cold Spring Harb Perspect Biol 2(4):a001040. https://doi. org/10.1101/cshperspect.a001040

38. Gottlieb E (2011) p53 guards the metabolic pathway less travelled. Nat Cell Biol 13(3):195-197. https://doi.org/10.1038/ncb2177

39. Hubackova S, Davidova E, Boukalova S, Kovarova J, Bajzikova M, Coelho A, Terp MG, Ditzel HJ, Rohlena J, Neuzil J (2020) Replication and ribosomal stress induced by targeting pyrimidine synthesis and cellular checkpoints suppress p53-deficient tumors. Cell Death Dis 11(2):110. https://doi.org/10.1038/s41419-0202224-7

40. Hu J, Locasale JW, Bielas JH, O'Sullivan J, Sheahan K, Cantley LC, Heiden MGV, Vitkup D (2013) Heterogeneity of tumorinduced gene expression changes in the human metabolic network. Nat Biotechnol 31(6):522-529. https://doi.org/10.1038/nbt.2530

41. Vander Heiden MG, DeBerardinis RJ (2017) Understanding the intersections between metabolism and Cancer biology. Cell. 168(4):657-669. https://doi.org/10.1016/j.cell.2016.12.039

42. Peng X, Chen Z, Farshidfar F, Xu X, Lorenzi PL, Wang Y et al Molecular characterization and clinical relevance of metabolic 
expression subtypes in human cancers. Cell Rep 23(1):255-69 e4. https://doi.org/10.1016/j.celrep.2018.03.077

43. Takeuchi T, Tomida S, Yatabe Y, Kosaka T, Osada H, Yanagisawa K, Mitsudomi T, Takahashi T (2006) Expression profile-defined classification of lung adenocarcinoma shows close relationship with underlying major genetic changes and clinicopathologic behaviors. J Clin Oncol 24(11):1679-1688. https://doi.org/10.1200/JCO. 2005.03.8224

44. Wang S, Liu F, Wang Y, Fan W, Zhao H, Liu L et al (2019) Integrated analysis of 34 microarray datasets reveals CBX3 as a diagnostic and prognostic biomarker in glioblastoma. J Transl Med 17(1):179. https://doi.org/10.1186/s12967-019-1930-3

45. Wei YT, Luo YZ, Feng ZQ, Huang QX, Mo AS, Mo SX (2018) TK1 overexpression is associated with the poor outcomes of lung cancer patients: a systematic review and meta-analysis. Biomark Med 12(4):403-413. https://doi.org/10.2217/bmm-2017-0249

46. Wu Y, Jamal M, Xie T, Sun J, Song T, Yin Q, Li J, Pan S, Zeng X, Xie S, Zhang Q (2019) Uridine-cytidine kinase 2 (UCK2): a potential diagnostic and prognostic biomarker for lung cancer. Cancer Sci 110(9):2734-2747. https://doi.org/10.1111/cas.14125

47. Zhu CM, Lian XY, Bi YH, Hu CC, Liang YW, Li QS (2018) Prognostic value of ribonucleotide reductase subunit M1 (RRM1) in non-small cell lung cancer: a meta-analysis. Clin Chim Acta 485: 67-73. https://doi.org/10.1016/j.cca.2018.05.042

48. Li L, Ng SR, Colon CI, Drapkin BJ, Hsu PP, Li Z et al (2019) Identification of DHODH as a therapeutic target in small cell lung cancer. Sci Transl Med 11(517). https://doi.org/10.1126/ scitranslmed.aaw7852

49. Liu Y, Marks K, Cowley GS, Carretero J, Liu Q, Nieland TJ et al (2013) Metabolic and functional genomic studies identify deoxythymidylate kinase as a target in LKB1-mutant lung cancer. Cancer Discov 3(8):870-879. https://doi.org/10.1158/2159-8290. CD-13-0015

50. McLarnon JG (2017) Roles of purinergic P2X7 receptor in glioma and microglia in brain tumors. Cancer Lett 402:93-99. https://doi. org/10.1016/j.canlet.2017.05.004

51. Burnstock G, Knight GE (2018) The potential of P2X7 receptors as a therapeutic target, including inflammation and tumour progression. Purinergic Signal 14(1):1-18. https://doi.org/10.1007/s11302017-9593-0

Publisher's note Springer Nature remains neutral with regard to jurisdictional claims in published maps and institutional affiliations. 